\title{
Auditory Nerve Fiber Responses to Combined Acoustic and Electric Stimulation
}

\author{
Charles A. Miller, ${ }^{1,2}$ Paul J. Abbas, ${ }^{1,2}$ Barbara K. Robinson, ${ }^{1}$ Kirill V. Nourski, ${ }^{3}$ Fahen Zhang, ${ }^{4}$ \\ AND Fuh-Cherng JenG ${ }^{5}$ \\ ${ }^{1}$ Department of Otolaryngology - Head and Neck Surgery, University of Iowa Hospitals and Clinics, Iowa City, IA 52242, USA \\ ${ }^{2}$ Department of Communication Sciences and Disorders, University of Iowa, Iowa City, IA 52242, USA \\ ${ }^{3}$ Department of Neurosurgery, University of Iowa Hospitals and Clinics, Iowa City, IA 52242, USA \\ ${ }^{4}$ Department of Communication Sciences and Disorders, University of Cincinnati, Cincinnati, OH 45221, USA \\ ${ }^{5}$ School of Hearing, Speech and Language Sciences, Ohio University, Athens, OH 45701, USA
}

Received: 1 June 2008; Accepted: 19 December 2008; Online publication: 10 February 2009

\begin{abstract}
Persons with a prosthesis implanted in a cochlea with residual acoustic sensitivity can, in some cases, achieve better speech perception with "hybrid" stimulation than with either acoustic or electric stimulation presented alone. Such improvements may involve "across auditory-nerve fiber" processes within central nuclei of the auditory system and within-fiber interactions at the level of the auditory nerve. Our study explored acoustic-electric interactions within feline auditory nerve fibers (ANFs) so as to address two goals. First, we sought to better understand recent results that showed non-monotonic recovery of the electrically evoked compound action potential (ECAP) following acoustic masking (Nourski et al. 2007, Hear. Res. 232:87-103). We hypothesized that post-masking changes in ANF temporal properties and responsiveness (spike rate) accounted for the ECAP results. We also sought to describe, more broadly, the changes in ANF responses that result from prior acoustic stimulation. Five response properties - spike rate, latency, jitter, spike amplitude, and spontaneous activity-were examined. Post-masking reductions in spike rate, within-fiber jitter and acrossfiber variance in latency were found, with the changes in temporal response properties limited to ANFs with high spontaneous rates. Thus, our results suggest how
\end{abstract}

Correspondence to: Charles A. Miller · Department of Otolaryngology Head and Neck Surgery - University of Iowa Hospitals and Clinics Iowa City, IA 52242, USA. Telephone: +1-319-3846758; fax: +1-3193846758; email: charles-miller@uiowa.edu non-monotonic ECAP recovery occurs for ears with spontaneous activity, but cannot account for that pattern of recovery when there is no spontaneous activity, including the results from the presumably deafened ears used in the Nourski et al. (2007) study. Finally, during simultaneous (electric+acoustic) stimulation, the degree of electrically driven spike activity had a strong influence on spike rate, but did not affect spike jitter, which apparently was determined by the acoustic noise stimulus or spontaneous activity.

Keywords: auditory nerve, cochlear implant, cat, single fiber, residual hearing, electric stimulation, acoustic stimulation, spontaneous activity, stochastic resonance

\section{INTRODUCTION}

\section{Background}

Persons with limited acoustic sensitivity who use an auditory prosthesis can demonstrate improved speech perception due to synergistic effects of combined acoustic and electric stimulation (Kiefer et al. 1998; von Ilberg et al. 1999; Gantz and Turner 2003; Cullen et al. 2004; James et al. 2005). The mechanisms of such enhancements and the auditory system's response to "hybrid" stimulation are not understood. Functional interactions of combined stimulation occur at central sites, as indicated by dichotic (Kong et al. 2005) and inferior colliculus 
(Snyder et al. 2004; Stakhovskaya et al. 2008) studies. Interactions also occur within auditory nerve fibers (ANFs). The histology of implanted cochleae indicates that inner hair cells (IHCs) can survive in regions apical, yet close to, the electrode array (Shepherd et al. 1995), raising the possibility that some ANFs can be excited by acoustic and electric stimuli. Such interactions could produce "enhanced" responses as well as interfering effects. Physiologic studies of peripheral and central neural responses are needed to understand functional acoustic-electric interactions.

Moxon (1971) studied ANF responses to combined electric and acoustic stimuli and described the electrophonic (“ $\beta$ ") response evoked by relatively low-level electric stimuli. This response is attributed to electrically driven contractile responses of outer hair cells that, in turn, generate a traveling wave and subsequent transduction by inner hair cells. With sinusoidal electric stimuli, $\beta$ responses were large for ANFs with best frequencies equal to the stimulus frequency. Furthermore, within-fiber acoustic-electric interactions were highly sensitive to the electric and acoustic stimulus frequencies (and relative phase) for responses mediated by the electrophonic mechanism. In contrast, broad (across-fiber) ANF excitation was evoked by higher-level electric stimuli that presumably directly depolarized ANF membranes (Moxon's “ $\alpha$ response"). While $\alpha$ responses are evoked from deaf ears (van den Honert and Stypulkowski 1984; Miller et al. 1999a), $\beta$ responses only occur in acoustically sensitive ears. Thus, for ears with only low-frequency residual hearing, within-fiber acoustic-electric interactions mediated by $\beta$ responses may only occur for low-frequency electric stimuli. von Ilberg et al. (1999) showed that, with low-rate periodic electric stimulation, ANFs can retain their acoustic tuning properties, suggesting that independent acoustic and electric encoding is also possible for some stimulus conditions, such as those avoiding the aforementioned acoustic interactions with $\beta$ responses. These findings indicate that, through the $\beta$ and $\alpha$ mechanisms, electric stimulation can excite ANFs in highly placespecific and non-place-specific manners and interactions depend upon level and frequency. However, even without acoustic stimulation, electrically evoked responses from acoustically sensitive ANFs likely differ from those of deaf ears, as inner hair cells produce spontaneous activity and, in some cases, may be depolarized by electric stimuli and produce $\delta$ responses (van den Honert and Stypulkowski 1984).

Nourski et al. (2007) used the electrically evoked compound action potential (ECAP) to study the influence of prior acoustic stimulation on ensemble responses to electric pulse trains. That study focused on the short-latency ECAP that is presumed to arise from $\alpha$ responses. Forward masking was provided by wide-band acoustic noise to approximate the rich spectral content of speech and environmental sounds, while the electric stimulus simulated the pulse-coding used by many auditory prostheses. Unexpectedly, in some cases, ECAPs evoked immediately after noise offset were larger than baseline values and their postmasking recovery was non-monotonic. The magnitude of the non-monotonic portion of the recovery curve (i.e., the decrease in ECAP amplitude that preceded the expected, subsequent, recovery of the ECAP) could be as large as $25 \%$ of the unmasked amplitude. Nourski et al. (2007) speculated that forward masking could transiently reduce the spontaneous rates (SRs) of ANFs and result in non-monotonic recovery. However, in further experiments using electric forward maskers, they reported non-monotonic functions from chemically deafened ears, suggesting that IHC activity was not essential to that result. A difficulty with ECAPbased measures is that they cannot distinguish among the multiple ANF response properties, described below, that can influence the ECAP. Furthermore, that study made reasonable inferences about the presence or absence of spontaneous activity in their normalhearing and chemically deafened ears, but did not actually measure spontaneous activity in any animals.

\section{Goals and rationales}

Two research goals were identified. First, we sought ANF data to understand the aforementioned ECAP results, hypothesizing that ANF responses undergo post-masking changes in temporal properties (i.e., jitter and latency), response rate, and spike amplitude, all of which influence ECAP amplitude (Miller et al. 1999b). We hypothesized that, after masker offset, the influence of spike-rate and amplitude decreases could be offset by reductions in withinand across-fiber temporal uncertainty. Furthermore, if those post-masking changes recovered at different rates, non-monotonic ECAP recovery could result. Masking-recovery data obtained by electrical stimulation of the cochlea provides some basis for this hypothesis. Miller et al. (2001) reported ANF threshold, jitter, and spike amplitude reductions immediately following masking. However, those data were obtained under different conditions, i.e., using single, electric, masking pulses and chemically deafened ears. The results obtained from hearing ears stimulated by relatively long-duration (400 ms) acoustic maskers, as in the Nourski et al. (2007) study, could differ substantially. Clearly, ANF measures are needed to identify the specific ANF response properties that underlie changes in the gross potential.

In addition to examining the ANF measures described above, we also sought to determine the 
extent to which each fiber's SR influenced the postmasker responses. The second goal was to follow-up on an earlier study of electrically excited ANFs with acoustic sensitivity (Miller et al. 2006). Thus, we sought ANF responses to simultaneous acoustic and electric stimulation, as well as those to electric stimuli following acoustic masking. While some simultaneous effects of electric and acoustic stimulation have been reported, post-acoustic effects on electric responses have not. Due to the different modes of ANF excitation for acoustic and electric stimulation, unique patterns of ANF recovery from prior stimulation may be expected.

To address these issues, we used a stimulus paradigm similar to that of the ECAP study. Responses were recorded for three stimulus conditions: (1) a $250 \mathrm{pulse} / \mathrm{s}$ electric train ("electric-only" stimulus), (2) a wideband acoustic noise ("acoustic-only" stimulus), and (3) the combined ("electric+acoustic") stimulus. By presenting each stimulus alone and simultaneously, acoustic-electric interactions could be assessed against baseline responses. The paradigm also provided for analysis of non-simultaneous effects, including the recovery of ANF properties following acoustic masking. An important contribution of this study is its assessment of aforementioned ANF response properties related to neural coding and the ECAP findings. The general approach of this work was to evaluate overall trends observed across a group of electrically and acoustically sensitive fibers. To that end, we often pooled data across ANFs and examined response properties as functions of the stimulusdriven rate (usually, the electrically driven rate).

\section{METHODS}

\section{Animal model and preparation}

Adult cats with normal hearing (assessed by the acoustic-click-evoked CAP) were used in acute, nonsurvival experiments. Details of surgical preparations are provided in Miller et al. (2006), a related study that characterized responses to electric stimuli from acoustically sensitive ANFs. Key methodology is described here. All procedures were conducted with animals at surgical levels of anesthesia with the approval of the University of Iowa Institutional Animal Care and Use Committee and in accord with regulations of the U.S. National Institutes of Health.

Using a surgical drill and rongeurs, a defect was made in the temporal bone overlying the left vestibulocochlear nerve. After exposure of the nerve, the bulla was opened to access the cochlea. A Pt/Ir ball electrode (diameter, $0.4-0.5 \mathrm{~mm}$, made from $3 \mathrm{mil}$ wire) was positioned on the bony margin of the round window (RW) to record the compound action poten- tial (CAP) for assessing acoustic sensitivity at various times during the experiment. A cochleostomy was made 1-2 $\mathrm{mm}$ medial to the RW using a $0.5 \mathrm{~mm}$ diamond burr. A second Pt/IR ball electrode (0.4$0.5 \mathrm{~mm}$ diameter) was inserted into the defect to a depth of about $1.5 \mathrm{~mm}$, providing for monopolar intracochlear stimulation. Standard micropipette techniques were used to record ANF potentials (Miller et al. 1999a or Zhang et al. 2007).

\section{Stimuli}

All stimuli were generated by Labview-based software that controlled an Instrutech Corporation ITC-18 data acquisition board, with 16-bit representation and $100,000 \mathrm{sample} / \mathrm{s}$ resolution. Stimulus delivery and response recording were controlled by Labview routines that were developed by our research group.

Acoustic stimuli Acoustic clicks were used to assess hearing sensitivity and were generated by low-rate (30 pulse/s) $100 \mu \mathrm{s} /$ phase biphasic pulses delivered to a Beyerdynamics DT48 earphone coupled to the external canal by a speculum. Acoustically evoked CAPs were recorded before and after the cochleostomy and insertion of the stimulating electrode to assess surgical and insertion trauma. For experimental data collection, the wideband acoustic noise was produced by a Grason-Stadler model 455C noise generator and gated by a Wilsonics electronic switch using a $1 \mathrm{~ms}$ rise-fall time. Together with the earphone and speculum, it produced within-canal spectra that were typically flat within $\pm 12 \mathrm{~dB}$ over $100 \mathrm{~Hz}$ to $30 \mathrm{kHz}$, with the exception of a $16 \mathrm{~dB}$ notch at $26 \mathrm{kHz}$. This calibration was based on two sets of measures: (1) the spectrum of the noise generator output (under load) as measured by a Stanford Research spectrum analyzer (model SR760) and (2) the levels of acoustic sinusoids (produced by a Tenma model 72-5015 function generator and earphone) measured using a withinspeculum probe tube mated to a Larson Davis 1/4" condenser microphone (model 2530) and sound level meter (model 824). Three noise-burst durations (100, 200, and $300 \mathrm{~ms}$ ) were used, with most data collected using $300 \mathrm{~ms}$ bursts. The use of the other durations is specified in the text. The acoustic level was adjusted to a level between 70 and $100 \mathrm{~dB}$ SPL as noted below. The acoustic-only stimulus consisted of the noise burst preceded by a $50-\mathrm{ms}$ silent period and followed by at least a 100 -ms silent interval. The pre-masker silent interval provided epochs for estimating SR and the second silent interval was used to assess post-masker SR reductions and recovery.

Electric stimuli All electric stimuli consisted of $40 \mu \mathrm{s} /$ phase rectangular biphasic pulses fed to a custom- 
made, battery-powered, current source having optical isolation and capacitive coupling. The current source's low-pass characteristic resulted in temporal "smearing" such that the pulses delivered to the cochlea had rise/fall times of approximately $10 \mu \mathrm{s}$. Low-rate (30 pulse/s) pulses were used to obtain ECAPs for estimating each nerve's dynamic range and a low-rate (10 pulse/s) search stimulus was set at a level that evoked an ECAP amplitude $85-90 \%$ of the maximum ("saturation") amplitude. For data collection, the electric-only stimulus consisted of a $250 \mathrm{pulse} / \mathrm{s}$ train with a typical duration of $500 \mathrm{~ms}$. While that rate is not as fast as the per-channel rates of some speech processors, the 4-ms inter-pulse interval allowed us to capture $\alpha$ and longer-latency $\beta$ responses (Javel and Shepherd 2000). For electric+acoustic stimulation, the acoustic noise was turned on $50 \mathrm{~ms}$ after the electric-train onset. This delay provided time for any electric-onset effects (i.e., modest degrees of adaptation) to occur prior to the masker onset (Zhang et al. 2007). A silent interval of $1,000 \mathrm{~ms}$ separated each of the three stimuli (as defined above) in an effort to minimize long-term adaptation while addressing the constraint of limited fiber-contact time (data collection for a single combination of stimulus levels required 2-4 min).

Upon contacting a fiber, the initial electric level was chosen to evoke a firing efficiency (FE) between $50 \%$ and $100 \%$ (as assessed over the first $50 \mathrm{~ms}$ of the electric pulse-train epoch). FE was computed as the ratio of the number of spikes evoked and electric pulses presented. Most frequently, four levels were examined for each fiber (typically $\pm 1 \mathrm{~dB}$ relative to the initial level) so as to obtain a range of electrically driven response rates. Across ANFs, threshold, defined as the level evoking an $\mathrm{FE}$ of $50 \%$ to the first pulse, was between 0.3 and $2.0 \mathrm{~mA}$, with a median value of $1.2 \mathrm{~mA}$. So that electric-acoustic interactions could be presumed to be assessed, the acoustic noise level was adjusted (within a $30 \mathrm{~dB}$ range) to evoke a strong response as assessed by visual inspection of the post-stimulus-time histogram (PSTH). We used 30 to 60 repeated stimulus presentations to obtain PSTHs and response statistics, with the repetition number adjusted on the basis of the electrically driven rate. Three stimulus parameters, electric level, acoustic level, and acoustic noise duration were explored as fiber-contact time permitted, with the highest priority given to exploring multiple electric levels.

\section{Response recording and data analysis}

Acoustic sensitivity of each nerve was assessed by clickevoked CAP using the RW electrode. CAPs were amplified $(100 \times)$ and low-pass filtered $(30 \mathrm{kHz}$, $120 \mathrm{~dB} /$ decade). All digital-to-analog conversions occurred at a 100,000 sample/s rate. ECAPs were recorded using two $\mathrm{Pt} / \mathrm{Ir}$ ball electrodes, with one positioned on the nerve trunk and the other $2 \mathrm{~mm}$ above the nerve, with both bathed in $0.9 \%$ saline solution. ECAPs were recorded in the same manner used to collect CAPs. Single-fiber recordings were amplified $(10 \times)$ and low-pass-filtered $(10 \mathrm{kHz}$ cutoff; $40 \mathrm{~dB} /$ decade) by an Axon Instruments Axoprobe headstage and amplifier, followed by additional filtering using Butterworth filters $(100 \mathrm{~Hz}$ high-pass, $40 \mathrm{~dB}$ /decade and $20 \mathrm{kHz}$ low-pass, $120 \mathrm{~dB} /$ decade). Electric noise synchronized to the power-line $(60 \mathrm{~Hz})$ frequency was reduced by a HumBug subtraction circuit (Quest Scientific Instruments, Inc., North Vancouver, Canada).

ANF responses were analyzed after data collection using custom Matlab (Natick, MA, USA; version 6.1) routines. They performed waveform "template" subtraction to reduce stimulus and ECAP artifacts (Miller et al. 1999a). Additional details concerning spikepicking are described in Miller et al. (2006). Three spike-timing statistics were measured relative to each electric pulse: (1) mean spike latency (measured from pulse onset to spike peak), (2) jitter, i.e., the standard deviation of spike times, and (3) vector strength (VS), measured with respect to the 4-ms period defined by the electric pulse rate. VS describes synchrony by expressing each spike's latency as an angle (between 0 and $2 \pi$ radians, mapped across the 4 -ms post-pulse response period), geometrically averaging those vectors, and computing the resultant vector's magnitude, normalized by the number of spikes examined. While jitter and VS reflect temporal uncertainty of spike times, they are not linearly related, justifying the report of both. There is, however, a degree of arbitrariness in the 4-ms period for VS computation; other periods would produce different VS distributions. Spike amplitude was measured from the peak to the following trough of the action potential. Each fiber's SR was computed by using the 50 -ms silent intervals that preceded each acoustic-only stimulus presentation. For a given fiber, this typically yielded $3 \mathrm{~s}$ of recording time for SR estimation.

Response characteristics were usually analyzed by pooling data across ANFs and across six selected time intervals chosen to describe key epochs before, during, and after the acoustic masker. This was done not only to describe general trends, but to also reduce the variability inherent in statistics based upon a limited number of stimulus repetitions (sweeps). Analysis intervals 1 and 2 covered $0-20 \mathrm{~ms}$ and 20$50 \mathrm{~ms}$ epochs after the electric train onset, providing baseline measures of conditions prior to masker onset. Intervals 3 and 4 spanned the first $30 \mathrm{~ms}$ and the last $20 \mathrm{~ms}$ relative to the noise-masker duration. Interval 5 covered the $20 \mathrm{~ms}$ immediately after masker 
offset. Interval 6 covered the last $100 \mathrm{~ms}$ of the electric pulse train, corresponding to the steady-state region of the electric response (Zhang et al. 2007). Interval 5 was used to assess post-masking effects and covers the period over which "enhanced" ECAPs were reported (Nourski et al. 2007). Some ANF responses were analyzed using per-pulse (i.e., $4 \mathrm{~ms}$ ) analysis bins to provide greater temporal detail of masking and postmasking trends.

As we sought to determine the effect of acoustic noise on electrically evoked responses, the dependent variables of several plots were measures obtained with "electric+acoustic" stimulation relative to "electriconly" measures. This normalization also reduced the bias introduced by the relatively small degrees of rate adaptation that can accompany 250 pulse/s electric trains (Zhang et al. 2007). Normalization to "electriconly" responses can be facilitated through the use of ratios (i.e., "electric+acoustic" data divided by "electric-only" data) or differences between the two measures. Each normalization provides unique information and also, of course, obscures an aspect of the neural responses. As it is not always clear (such as in the case of jitter measures) as to which normalization method provides the better assessment relative to information coding or the ECAP, both normalizations were used in some data presentations. Finally, SRs were subtracted from "raw" spike rates when reporting acoustically or electrically driven rates, using the formula of van den Honert and Stypulkowski (1984).

Statistical tests were typically limited to relatively straightforward $t$-tests applied across two conditions or tests of the significance of a linear correlation (Bevington and Robinson 2002). Bonferroni corrections were used to avoid claims of statistical significance due to the use of multiple or correlated tests. We generally used a conservative correction factor, typically equal to the number of plots shown in each relevant figure. Trends are often described by median values to avoid bias from outlier data.

\section{RESULTS}

\section{Data set and overview}

Data were obtained from 78 fibers of 13 cats. All but one fiber provided data within our target of electrically evoked FEs of at least $50 \%$ (see the "Acoustic stimuli" sub-section under Methods). The data from those 77 fibers were typically analyzed for across-fiber (group) trends. In the one other case, only low-FE responses (again, to the electric-only stimulus) were obtained and provided an atypical data set, described at the end of this section. Losses in acoustic sensitivity ranging from $10-15 \mathrm{~dB}$ were noted immediately after the cochleostomy and electrode insertion. Additional losses were typically noted over the 12-18-h datacollection period. Each experiment was stopped if CAP threshold increased by more than $40 \mathrm{~dB}$ relative to the initial post-surgery value. On average, acoustic threshold elevations for the ANFs (relative to published normative data) was $26 \mathrm{~dB}$. Greater details of losses encountered with this preparation was described in Miller et al. (2006). Spikes evoked by the electric pulses were typically short-latency $\alpha$ responses. Electrophonic $(\beta)$ responses were observed in 18 (23\%) fibers, although they were typically not observed in the post-masking analysis windows, most likely because $\beta$ responses adapt faster than do $\alpha$ responses (Zhang et al. 2007).

After presenting example PSTHs, the "General group trends" sub-section summarizes how combined (electric+acoustic) alters ANF responses to electric stimuli. Electric-level effects and the additivity of acoustic and electric responses are given additional attention in the subsequent two sub-sections. After a brief description of acoustic-duration effects, a detailed analysis of post-acoustic changes in ANF responses is provided in the large sub-section entitled "Post-masking changes in ANF responses". All of these analyses are based on the use of the six analysis intervals. In the subsequent sub-section, individual ANF data are shown using smaller time bins to show greater temporal detail. The final sub-section describes infrequently observed "enhanced" responses. In this report (and particularly in the figures), the three stimuli are also referred to by the letters "A", "E" and "EA". For brevity, the term "combined" is sometimes used to refer to simultaneous electric+acoustic stimulation.

\section{Data set and exemplar data}

Figure 1 presents PSTHs for an ANF excited by the three stimuli. A diagram of the electric and acoustic stimulus timing is shown at the top of the figure, while the six analysis windows are indicated at the bottom. With electric stimulation, the PSTH has regularly spaced peaks, reflecting synchronization to each pulse. Those peaks demonstrate variability in the number of spikes, likely due to randomizing effects of the significant spontaneous activity in this case. The use of wider, $40 \mathrm{~ms}$, bins (not shown) reveals modest (18\%) rate adaptation for electric-only stimulation, consistent with trends from deafened cats (Zhang et al. 2007). The middle ("acoustic-only") PSTH has a sharp onset peak typical of acoustic responses, followed by a rapid decay to a steady state. Spontaneous activity is reduced in the post-masking interval. The PSTH for combined (electric+acoustic) stimulation (bottom panel) lacks clear peaks in the response to the electric pulses during the noise burst and rate 


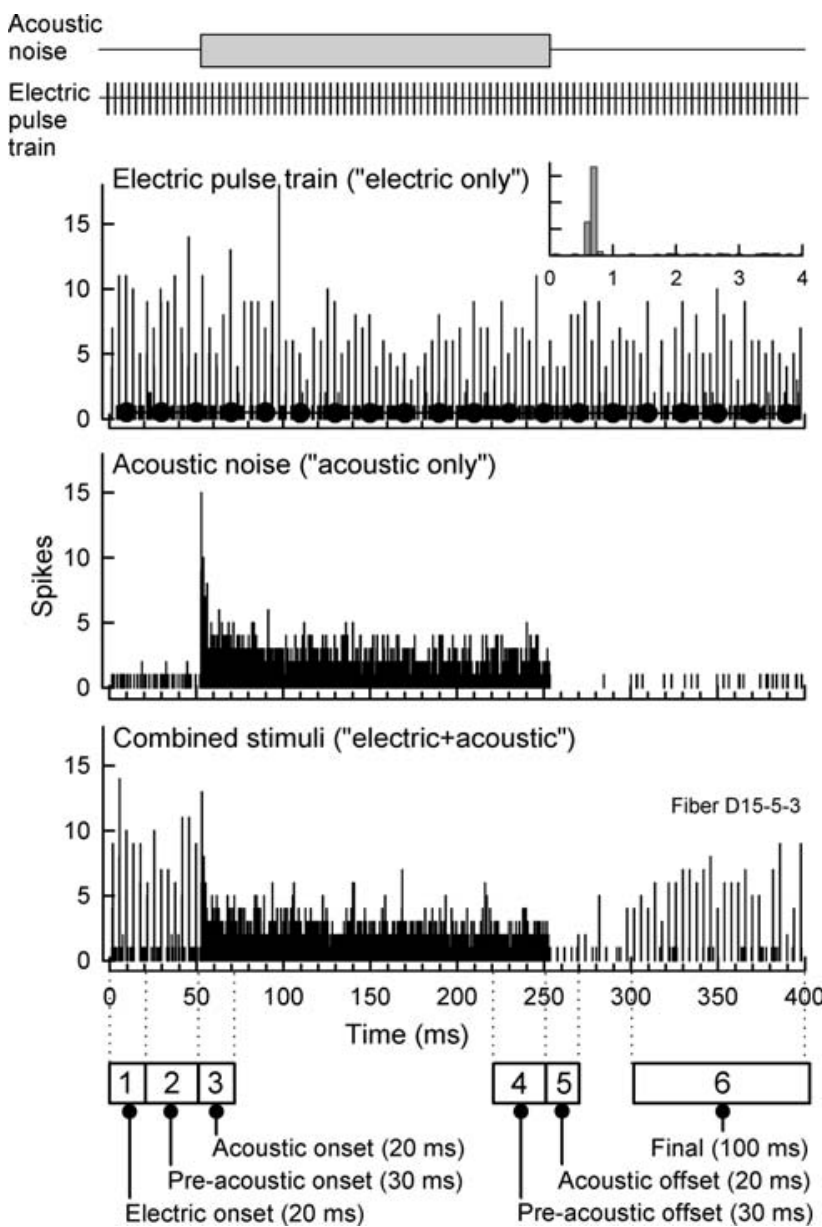

FIG. 1. Summary of key methods and exemplar data. The relative timing of the wideband acoustic noise and the 250 pulse/s electric train is shown schematically at the top. PSTHs from one ANF are shown in response to the electric train presented alone, the acoustic noise presented alone, and the combined stimuli. At the bottom are shown the six time intervals used to analyze ANF responses that were used in this study. The period histogram for the response to the 250 pulse/s electric-only stimulus is included within the top PSTH. Spanning a $4 \mathrm{~ms}$ period, it indicates highly synchronized, shortlatency responses consistent with $\alpha$ responses (vertical tick $=200$ spikes).

adaptation and recovery is evident across intervals 5 and 6.

These PSTHs demonstrate several response features. The post-noise reduction in responses locked to the electric pulses is, to a degree, analogous to acoustic noise masking of tone-burst responses (i.e., Kiang et al. 1965), as is the reduction in electrically evoked spike rates (Smith 1977). We note, however, that spike latencies for "electric-only" excitation were less than $1 \mathrm{~ms}$ (see the period histogram within Fig. 1), indicating direct depolarization of ANF membranes (i.e., $\alpha$ responses). Thus, the excitation mechanisms related to this study differ from the purely hair-cell-mediated responses reported in the earlier studies.

\section{General group trends}

In this paper, ANF responses are often characterized by their group, across-fiber, trends using the six analysis epochs. Post-masking interval 5 responses were given particular scrutiny so as to address the study's first goal. Spike rate, jitter, VS, and spike amplitude-ANF properties hypothesized to affect ECAP amplitude - were assessed in each interval. As the effect of the noise on electrically evoked responses is a key objective of this study, ANF response properties are frequently expressed as ratios of the values obtained under combined and electric-only stimulus conditions.

Figure 2 summarizes how acoustic noise modified ANF responses to electric pulses across the analysis intervals. As intervals 1 and 2 covered epochs preceding the masker, measures from them were combined. In the graphs in the figure's left column, the dependent variables were computed by subtracting "electriconly" measures from "electric+acoustic" measures. In the right column, ratios of the "electric+acoustic" and "electric-only" data are plotted. Individual and median data for 77 fibers are plotted for spike rate (Fig. 2A), jitter (Fig. 2B), VS (Fig. 2C), and amplitude (Fig. 2D). As each fiber was typically assessed at multiple electric levels (typically spanning ranges of $2 \mathrm{~dB}$ or less), each contributed more than one point to the plots.

With few exceptions, the addition of acoustic noise increased spike rate (Fig. 2A, intervals 3 and 4) and jitter (Fig. 2B, same intervals), while VS was reduced. In postmasking interval 5, both jitter and spike rate were reduced. This contrasts with the well-documented reciprocal relationship between these two properties in the input-output functions of electrically stimulated deaf fibers (Miller et al. 1999a). Unpaired Bonferronicorrected $t$-tests were performed for the four response measures to determine if the interval 5 ratios were statistically different from the pre-acoustic values (i.e., data from intervals 1 and 2 combined). A conservative, eightfold Bonferroni correction was used. Briefly summarizing the results, for spike rate, jitter, and VS, the "EA/E" ratios over the interval 5 responses were significantly different from the pre-masking ratios, with all corrected error probabilities less than 0.001 . The statistical comparison of the interval 5 spikeamplitude ratios and the amplitude ratios for intervals 1 and 2 failed to indicate any post-masking effect (uncorrected $p_{\text {error }}>0.5$ ). There is statistical evidence that the amplitude of spikes evoked with combined stimulation is reduced relative to pre-masking (interval 1 and 2) values. A corrected $t$-test (correction factor, 9 ) of the difference between interval 4 amplitude ratios and the ratios of intervals 1 and 2 indicates statistical significance $\left(t=3.00, p_{\text {error }, \text { corrected }}=0.024\right.$, 

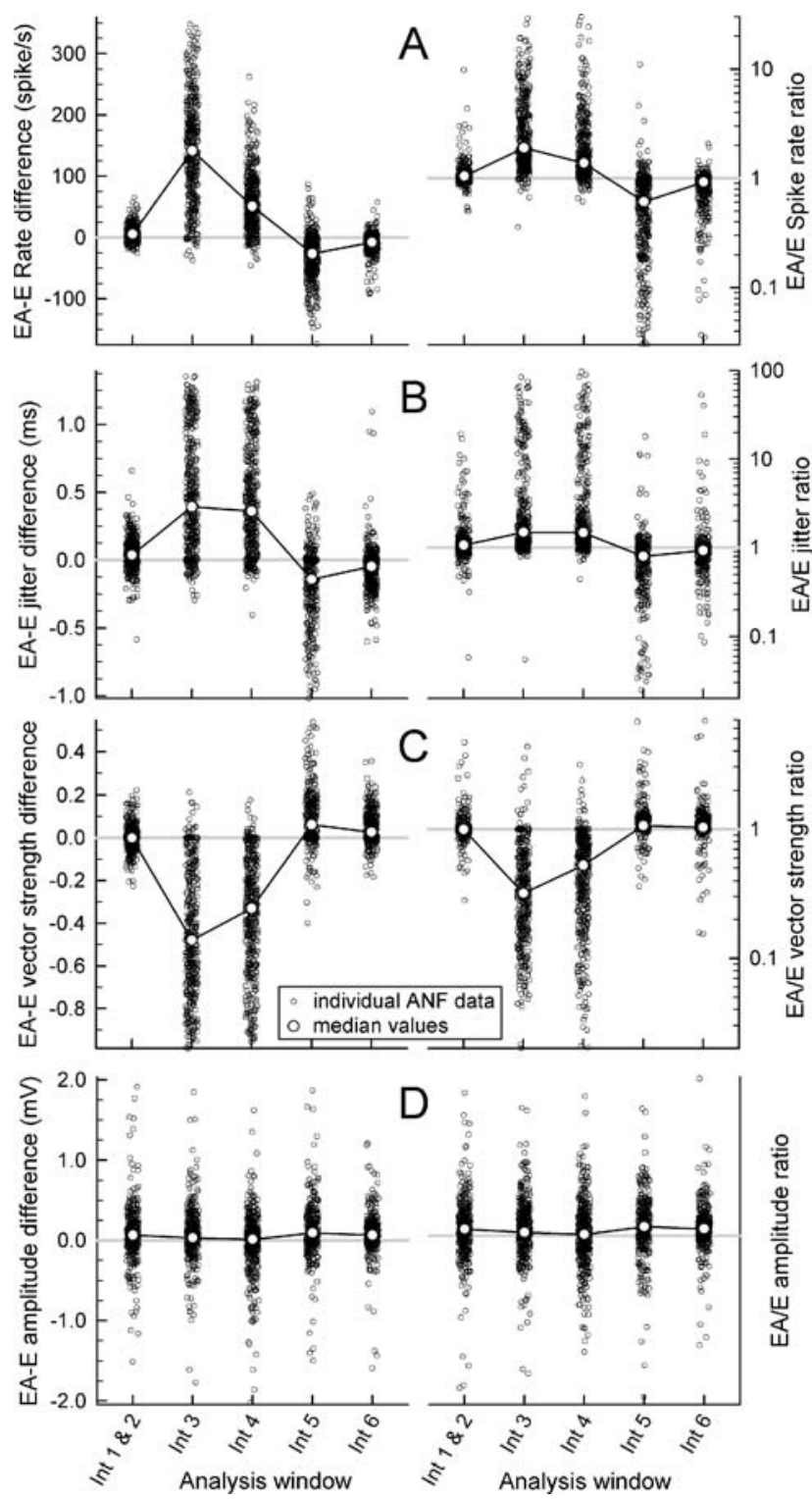

FIG. 2. The six-window analyses demonstrate that acoustic masking reduces electrically evoked spike rates (A) and temporal uncertainty (jitter, B and VS, C), while spike amplitude (D) is unchanged. In the left column, those measures are expressed as differences between the "electric+acoustic" responses and the "electric-only" responses while the right column expresses ratios of those two responses. Data from 77 ANFs (plotted with open circles) and median values (connected by line segments) are plotted as functions of the analysis window number. Data from intervals 1 and 2 were averaged together as both intervals covered pre-masking periods. A small amount of variability was added to the abscissa values to make the data more visible. The gray horizontal lines indicate conditions of no change between "electric+acoustic" and "electric-only" responses.

$\mathrm{df}=817)$, although the median amplitude change is very small $(\sim 1 \%)$.

Summarizing the analyses based on the Figure 2 data, acoustic forward masking decreases spike rate and spike-timing variability over the $20 \mathrm{~ms}$ epoch immediately following masker offset. However, no post-masking change in spike amplitude was found. With respect to the ECAP, the post-masking changes in spike rate and jitter would be expected to change ECAP amplitude in opposing directions.

Effect of electrically driven spike rate on simultaneous and post-masking responses

The summary plots of Figure 2 combined data across ranges of electrically driven spike rates, obscuring the effect of electric level. To assess the influence of the electrically driven rate on two measures with robust electric/acoustic interactions, EA/E ratios for spike rate and jitter are plotted in Figures 3-A, B versus the spike rates evoked by electric-only stimulation. These relationships were evaluated for two conditions: combined stimulation and post-acoustic electric stimulation. For combined stimulation, the dependent variables were measured over interval 4 to provide an assessment of the "steady state" responses to the two stimuli. Those data are plotted in the graphs within the left column of Figure 3. The post-acousticmasking effects on electric responses, based on interval 5 measures, are shown in the right column of Figure 3. Electrically driven spike rates (the independent variable) were computed over premasker intervals 1 and 2 (i.e., the first $50 \mathrm{~ms}$ of the electric train). That range of rates (up to $320 \mathrm{spike/s}$ ) exceeds the electric pulse rate due to spontaneous activity and $\beta$ responses that can be observed over this initial period (Miller et al. 2006).

Effect on spike rate The trend of normalized electric+ acoustic spike rates (Fig. 3-A) is clear and rather predictable. Across electrically driven rates, the addition of acoustically evoked activity to electrically evoked activity almost always results in higher spike rates. This increase becomes proportionally smaller as the electrically driven rate is increased, consistent with the greater influence of the electric stimulus at higher current levels. However, as driven rate is increased, the spike-rate ratios do not reach the asymptotic value of 1 . Additional analyses of the simultaneous addition of electric and acoustic spike activity are described in the sub-section entitled "Summation of acoustically evoked and electrically evoked spike activity", below. The electrically driven rate also strongly influences post-masking rate decrements (Fig. 3-E). At low and moderate driven rates, there is large variability in the degree of rate adaptation. High driven rates can greatly reduce the rate reductions caused by acoustic forward masking within the $20 \mathrm{~ms}$ post-noise epoch.

Effect on spike jitter The electrically driven rate also influences the "EA/E" jitter ratios during combined 


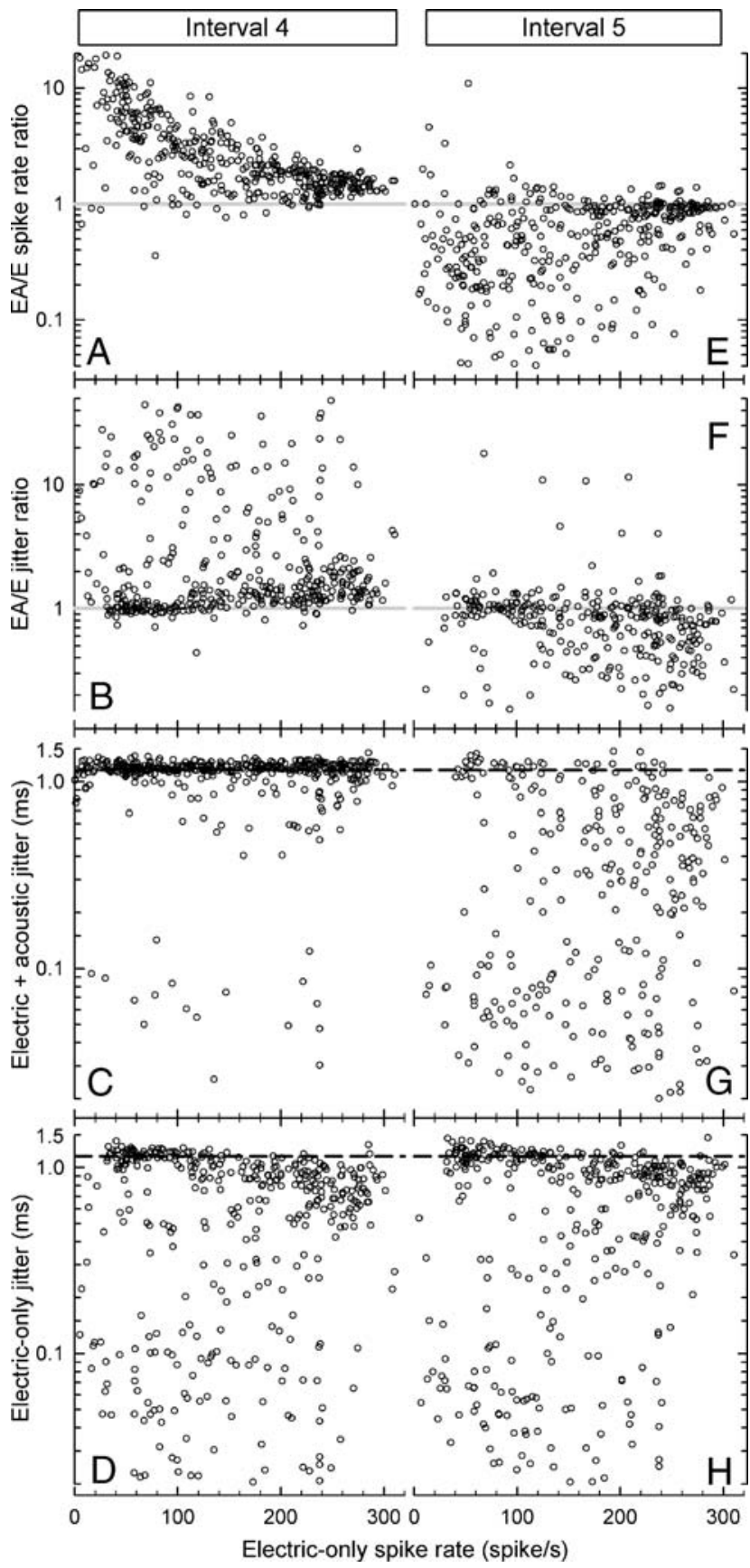

stimulation (Fig. 3-B), but in a way opposed to the trend observed for the rate ratios (Fig. 3-A). At low driven rates, most jitter ratios cluster near 1 . As driven rate is increased beyond $100 \mathrm{spike/s}$, there is a trend of modest increases in the ratios. Relative to the spikerate trends, this jitter-ratio trend is significantly different. The two plots of Figures 3-C, D show how electric+acoustic jitter and electric-only jitter change with the electrically driven rate and help explain the jitter-ratio trends. Jitter values for combined stimulation (Fig. 3-C) are narrowly distributed around the value $(1.15 \mathrm{~ms}$, dashed line) predicted by random spike
FIG. 3. The effects of electrically driven spike activity on spike rate and jitter measured during "electric+acoustic" stimulation (A and $\mathbf{B}$, respectively) and after masker offset (E and $\mathbf{F}$, respectively). Spike-rate and jitter are expressed as ratios ("electric+acoustic"/"electric-only") to indicate the effect of the acoustic stimulus, with the gray lines indicating no change $(r a t i o=1)$. For combined stimulation, the increase in spike rates becomes smaller as the electrically driven rate is increased $(\mathbf{A})$, yet remain greater than the "electric-only" rates. Post-masking rate recovery is increased by driving ANFs to higher rates $(\mathbf{E})$. During combined stimulation, higher electrically driven rates result in larger jitter ratios (B). As described in the text, the plots of absolute jitter for combined stimulation (C) and electric-only stimulation (D) aid in the interpretation of the jitter-ratio trend. In all plots, the abscissa indicates the rate evoked by electric-only stimuli over pre-masking intervals 1 and 2. Measures obtained with "combined" stimulation (A, B, C, and D) were assessed using interval 4; post-masking measures $(\mathbf{E}, \mathbf{F}, \mathbf{G}$, and $\mathbf{H}$ ) were assessed over interval 5. The dashed lines in $\mathbf{C}, \mathbf{D}, \mathbf{G}$, and $\mathbf{H}$ indicate the jitter expected by random spike timing. For each left-right pair of graphs, the ordinate axes have the same scaling.

timing over the $4 \mathrm{~ms}$ analysis window used for our jitter computation. Also, there is no indication of a dependence on the electrically driven rate. For electriconly stimulation (Fig. 3-D), the jitter values cluster around the "random value" for rates less than about $80 \mathrm{spike/s.} \mathrm{At} \mathrm{higher} \mathrm{rates,} \mathrm{electric-only} \mathrm{jitter} \mathrm{decreases}$ to values lower than those obtained with combined stimulation. This trend is consistent with the increased jitter-ratio trend shown in Figure 3-B.

Finally, in the post-masking epoch of interval 5, $\mathrm{EA} / \mathrm{E}$ jitter ratios cluster around 1 for low driven rates and for higher rates $(>100$ spike/s) there is a trend toward lower ratios as driven rate is increased. However, there is a significant amount of uncorrelated variability in the jitter ratios. A comparison of the electric+acoustic and electric-only jitter plots is revealing. There is little indication that the electric +acoustic jitter in the post-masking interval (Fig. 3-G) is correlated with the electrically driven rate and there is a large degree of uncorrelated variability in the jitter values. However, electric+acoustic jitter is typically lower than the value (dashed line) expected for random timing. In contrast, for electric-only stimulation over interval 5 , there is a downward trend as the electrically driven rate is increased (Fig. 3-H). It is interesting to note that the division of electric+acoustic jitter values by the electric-only values (i.e. the ratios of Fig. 3-F) results in less variation than is evident in either Figure 3-G or Figure 3-H. Typically, one would expect that the ratio of two variables, each of which is subject to a degree of uncorrelated variability, would have comparable, if not greater, levels of variability. The fact that the variability across the Figure 3-F ratios is relatively small suggests that the variability present in each of the two lower plots are not caused by a random process, but are due to some other process (e.g., acoustic driven rate, spontaneous rate, degree of rate adaptation, etc.) common to both electric+acoustic and electric- 
only jitter. Such possibilities are addressed later in this report.

Summation of acoustically evoked and electrically evoked spike activity

Combining acoustic and electric stimuli did not result in proportionally greater responses, i.e., those spike rates did not equal the summed rates for each stimulus presented alone. Figure 4 plots interval 4 spike-rate data for combined stimulation versus spike rates produced by each stimulus presented alone. The dependent variables are "electric+acoustic"/"electriconly" rate ratio (Fig. 4A) and the "electric+acoustic"/ "acoustic-only" rate ratio (Fig. 4B). The independent variables provide a means of assessing the influence of the effective electric and acoustic levels across a pool of data from 77 ANFs. Comparisons of Figure 4A, B trends are aided by parsing the data into subgroups defined by the acoustically and electrically driven rates, respectively, with an effort to match each group's median value across the two graphs. Those medians were then used to predict the rate ratios for linear addition of acoustic and electric spike rates (dashed lines). Figure $4 \mathrm{~A}$ covers a wider range of electrically driven rates $(0-350 \mathrm{spike/s})$ relative to the range of acoustically driven rates $(0-200 \mathrm{spike} / \mathrm{s}$, Fig. 4B). This is likely due to the experimental design, which used a relatively small range of acoustic levels. Generally, the data lie below the corresponding predicted curves, indicating less than linear summation. However, for the region of low electrically driven rates $(<50$ spike/s) and acoustically driven rates greater than 70 spike/s (gray circles and black dots within Figure 4A) the data group around their hyperbolic prediction lines, indicating nearly linear addition of spike activity. For those same two groups of acoustically driven rates, increases in the electrically driven rate result in spikerate ratios that deviate downward from the two corresponding prediction lines and approach a common asymptote. This is consistent with a firing process limited by refractory and adaptation-like processes and the dominance of the electric responses at high electric levels. Large deviations from the linear prediction also occurred for both low acoustically and low electrically driven rates, as shown with the open symbols in Figure 4A, B, respectively. This trend is consistent with nonlinear "thresholding" that is dominant at low levels.

\section{Noise-duration effects}

The effect of noise duration was examined by comparing the interval 5 measurement ratios obtained using maskers of 100 and 300 ms duration. Interval 5 spike-rate ratios and jitter ratios were
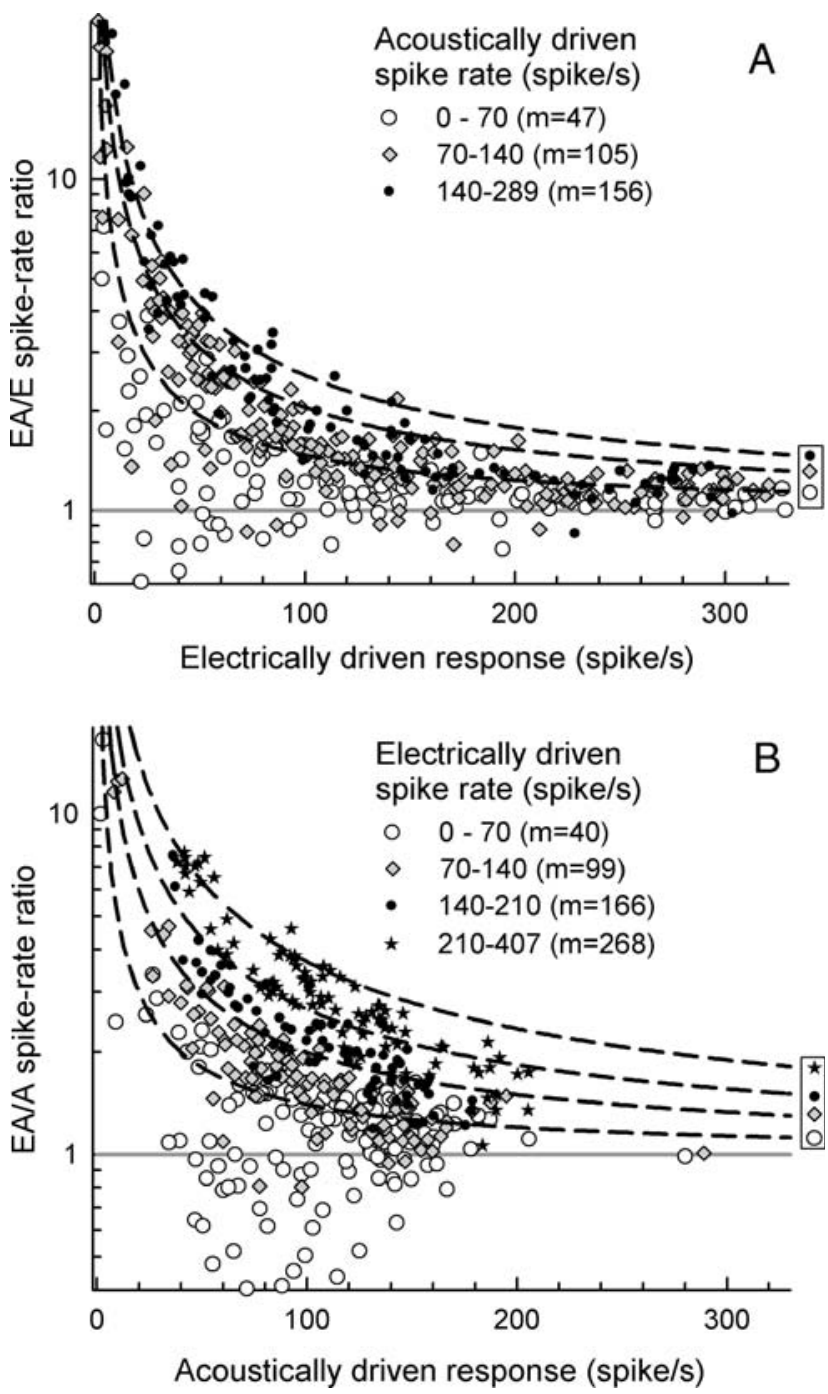

FIG. 4. The simultaneous addition of one stimulus (electric or acoustic) to the other generally results in spike rates less than predicted by addition of the rates evoked by each stimulus. Data from 77 fibers are pooled to show group trends and data were derived from analysis in interval 4 . In $\mathbf{A}$, ratios of spikes evoked by the combined stimuli and the electric-only stimulus are plotted vs. rates evoked by the electric-only stimulus. The parameter is the range of spike rates evoked by the acoustic stimulus, with median values for each data subset shown in parentheses. Hyperbolic curves (dashed lines) indicate ratios expected for each subset if the results of combined stimulation were additive (curves are identified with each subset by the boxed symbols at the right margin). These prediction curves were computed using the aforementioned median values, as indicated by the symbols, aligned with the curves, that are shown in the small rectangle at the right margin of the graph. B presents analogous data as a function of acoustically driven rate. In this case, the ordinate value is the rate obtained with combined stimulation, normalized to the acoustic-only condition. At low electric levels and relatively high acoustic levels (A), linear addition is indicated. The gray horizontal line indicates a ratio of 1 .

examined, again using the "E+A/E" ratio. For these comparisons, the 100 and $300 \mathrm{~ms}$ data sets were matched by fiber, and each fiber's electric level and acoustic level. For the interval 5 spike-rate ratio data, 
the median ratios for the 100 and $300 \mathrm{~ms}$ data sets were 0.65 and 0.33 , respectively, suggesting greater rate suppression with the longer noise burst. A corrected $t$-test of the interval 5 spike-rate ratios (adjusted for the use of two tests in this noise-duration analysis) indicated that the rate ratios were indeed generally smaller for the longer noise bursts $(t=2.9$, $\left.p_{\text {error,corrected }}=0.0085, \mathrm{df}=148\right)$. For the interval 5 jitter ratio data, the median ratios for the 100 and $300 \mathrm{~ms}$ data sets were 0.767 and 0.737 , respectively. The corrected $t$-test indicated that the jitter ratios were, on average, not significantly different $\left(t=0.164\right.$, $p_{\text {error, }}$ corrected $=0.92, \mathrm{df}=96)$. To summarize, an increase in the acoustic noise duration (from 100 to $300 \mathrm{~ms}$ ) resulted in greater post-masking spike-rate reductions, while no greater reduction in jitter was evident.

\section{Post-masking changes in ANF responses}

Changes in spike rate Detailed analyses of the ANF response properties that may relate to post-masking (i.e., interval 5) changes in spike rate were undertaken to address our hypothesis relative to the Nourski et al. (2007) data. In Figure 5, post-masking rate changes (i.e., the difference between the $\mathrm{E}+\mathrm{A}$ and E-only responses for interval 5) are plotted against two variables descriptive of the influence of the acoustic noise on ANF responses. The graphs of the left column plot rate ratios versus the acoustically driven rate as assessed across interval 3 (the acoustic-onset interval) while the right-column graphs plot the same data versus the rate reduction that occurred between intervals 3 and 4 for acoustic-only stimulation. Thus, Figure 5-A, C, E provide an assessment of the influence of the strength of the acoustic stimulus, while Figure 5$\mathrm{B}, \mathrm{D}, \mathrm{F}$ show how the degree of rate adaptation affects the post-masking changes in electrically driven rates. Data were obtained from 77 fibers and are divided into three groups according to SR. Group 1 (Fig. 5-A, B) contains ANFs with essentially no spontaneous activity (i.e., $0-0.65 \mathrm{spike} / \mathrm{s}$ ). The inclusion of data from fibers with non-zero SRs was done to increase the size of the Group 1 data set. Group 2 (Fig. 5-C, D) encompasses low-SR fibers $(0.65-15$ spike/s) while Group 3 includes high-SR fibers (SR>15 spike/s). Finally, for the high-SR plots, the data are subdivided into three groups according to interval 5 spike rates produced by the "electric-only" stimulus.

Across the six scatter plots, there is a general trend toward larger rate decrements with increases in either the acoustically driven rate or the acoustically driven rate decrement. Linear regressions were performed on each scatter plot (dotted lines). Tests of the significance of each regression were performed using the hypothesis that there was no statistically significant decrease in each of the scatter plots and Bonferroni
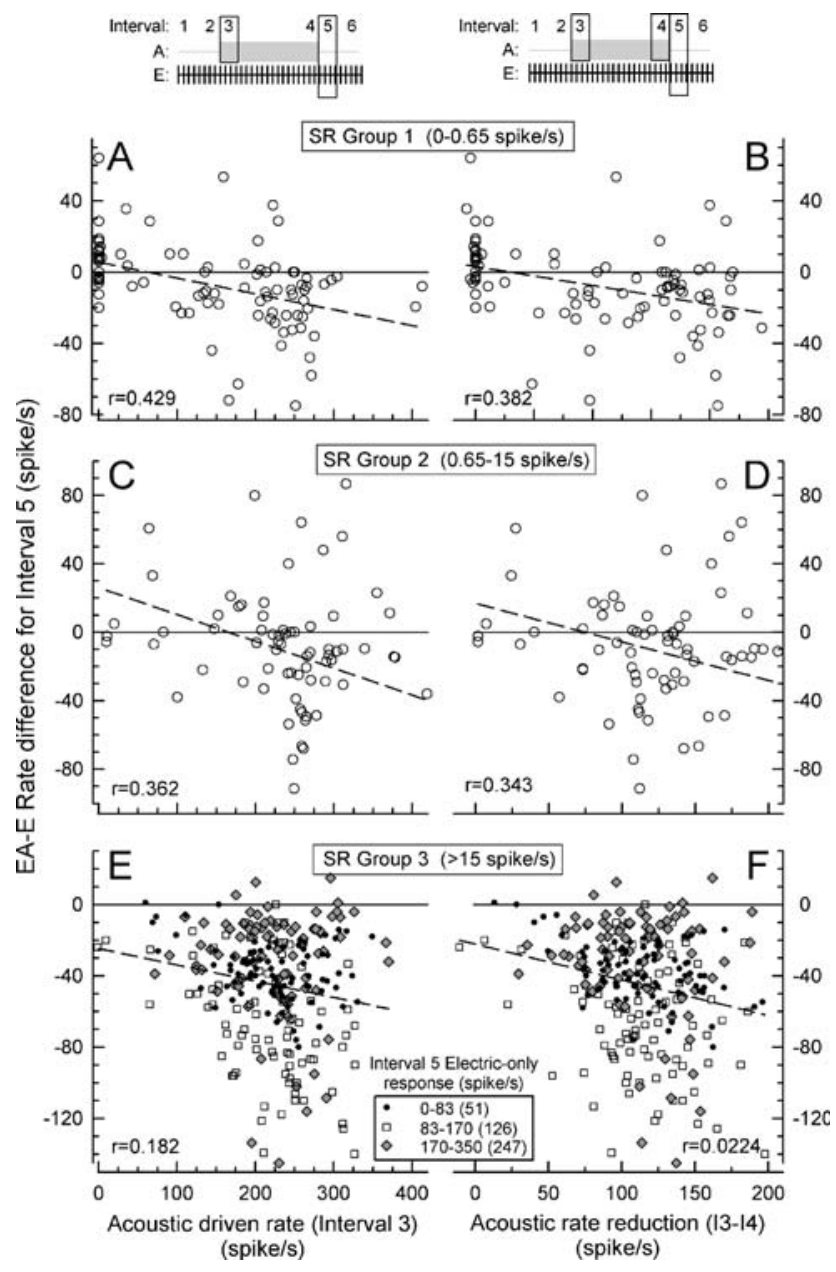

FIG. 5. The degree of acoustically evoked activity correlates with post-masking decreases in electrically evoked spike rates for all three SR groups. In all six plots, the dependent variable is the difference between spikes rates for the "electric+acoustic" and the "electriconly" conditions assessed in the post-masking interval 5 . In the left column (A, C, and $\mathbf{D})$, those differences are plotted versus the initial (interval 3) spike rate evoked by the acoustic stimulus, while the right column plots (B, D, and $\mathbf{F}$ ) the same data versus the rate decrement measured across intervals 3 and 4 for the acoustic-only condition. Linear regressions (dashed lines) produced low correlation coefficients, but were statistically significant. The diagram at the top provides a reminder of the analysis intervals used for the two columns of data. In $\mathbf{E}$ and $\mathbf{F}$, the different symbols indicate three ranges of spike rates evoked by electric-only stimulation within interval 5.

corrections were applied to account for the six data sets. Table 1 provides the results of these regressions and tests. Summarizing those results, we found significant decreasing trends for both the acoustically driven rate and rate decrement manipulations for all three SR groups. Comparisons among the three subgroups within Figures 5-E, F, which divide the data according to electrically driven rates, suggest that increases in the effective electric level reduce the magnitude of the rate decrements, as would be expected. Finally, we note that the relationships 
TABLE 1

\begin{tabular}{|c|c|c|c|c|c|}
\hline \multicolumn{6}{|c|}{ Summary of the statistical tests performed on the linear regressions of Figure 5} \\
\hline & Fiber SR group & & 1 & 2 & 3 \\
\hline SR range (spike/s) & & & $0-0.65$ & $0.72-12.2$ & $16.9-116$ \\
\hline SR median & & & 0.0 & 3.9 & 52.4 \\
\hline Number of fibers & & & 16 & 15 & 46 \\
\hline Observations & & & 91 & 74 & 260 \\
\hline $\begin{array}{l}\text { Linear regression } \\
\text { result }(r) \text { and statistical } \\
\text { significance }\end{array}$ & $\begin{array}{l}\text { I5 Rate difference } \\
\text { vs. I3 Acoustic rate } \\
\text { (Figs 4A, C, E) } \\
\text { I5 Rate difference } \\
\text { vs. (I4-I3) acou. rate } \\
\text { diff (Figs. 4B, D, F) }\end{array}$ & $\begin{array}{l}r \\
T \\
p_{\text {error, corrected }} \\
\mathrm{df} \\
r \\
T \\
p_{\text {error, corrected }} \\
\mathrm{df}\end{array}$ & $\begin{array}{l}0.429 \\
4.48 \\
<0.001 \\
89 \\
0.382 \\
3.90 \\
<0.001 \\
89\end{array}$ & $\begin{array}{l}0.362 \\
3.29 \\
0.0046 \\
72 \\
0.343 \\
3.10 \\
0.0084 \\
72\end{array}$ & $\begin{array}{l}0.181 \\
2.96 \\
0.0102 \\
258 \\
0.224 \\
3.69 \\
<0.001 \\
258\end{array}$ \\
\hline
\end{tabular}

These regressions were computed for plots of interval 5 spike-rate differences versus (1) acoustically driven rates within interval 3 and (2) changes in the acoustically driven rates across intervals 3 and 6 . As in other analyses, the interval 5 spike-rate differences were computed by subtracting "electric-only" rates from "electric+acoustic" rates. For the six scatter plots of Figure 5, the tests of the significance of the linear regressions addressed the hypothesis that the fitted slopes were not less than zero. In all six cases, the tests indicated statistically significant regression slopes at error probabilities of 0.01 or smaller, using Bonferroni-corrected probabilities

underlying the plots of Figure 5-E, F may not be best described by straight lines.

Reductions in interval 5 jitter due to prior acoustically driven activity Analyses of post-masking changes in jitter were conducted using the same approach used for spike-rate analyses of the previous sub-section. Figure 6 presents jitter differences (between "electric+ acoustic" and "electric-only" measures) for interval 5 plotted as functions of the acoustically evoked onset spike rate (Figs. 6-A, C, E) and versus the acoustically evoked rate decrement (Figs. 6-B, D, F). The data were again divided into the three SR groups and linear regressions were computed for each of the six scatter plots. There is a general trend toward greater interval 5 jitter decrements for higher SRs. For the Group 1 and Group 2 data sets, the tests of the significance of each linear regression indicated a lack of statistical significance (at $p_{\text {error }}=0.09$ or greater), even without Bonferroni corrections. However, both Group 3 linear regressions were statistically significant with the Bonferroni corrections. These results are summarized in Table 2. We note that, among the 12 tests of the linear regressions shown in Figures 5 and 6 , the number of data comprising the Group 2 jitter plots (i.e., Figs. 6-C, D) was the smallest, suggesting that relatively low statistical power may have influenced the null statistical results for those data sets. Consequently, we performed further analyses of possible jitter-SR relationships, as described below.

The influence of SR on post-masking changes in jitter is examined directly in Figure 7, which plots interval 5 electric+acoustic vs. electric-only jitter differences (Fig. 7A) and jitter ratios (Fig. 7B) as functions of

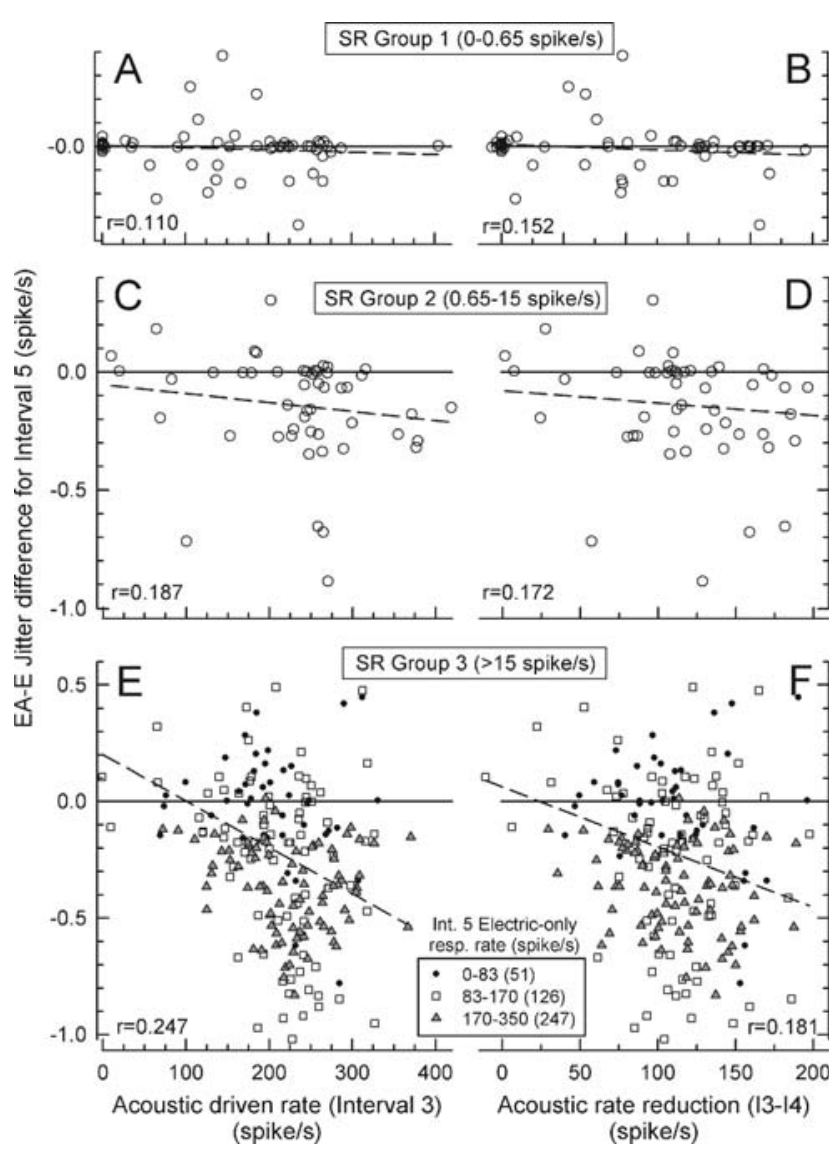

FIG. 6. Analyses of interval 5 responses indicate that the degree of acoustically driven activity influences post-masking jitter reductions. The plots provide the same analyses for jitter that Figure 5 provided for spike rate. In contrast to the Figure 5 trends, post-masking jitter changes are only evident for the Group 2 and 3 ANFs, i.e., fibers with significant spontaneous activity. 
TABLE 2

Summary of the statistical tests performed on the linear regressions of Figure 6, which concerned interval 5 jitter differences caused by acoustic forward masking

\begin{tabular}{|c|c|c|c|c|c|}
\hline & Fiber $S R$ group & & 1 & 2 & 3 \\
\hline SR range (spike/s) & & & $0-0.65$ & $0.72-12.2$ & 16.9-116 \\
\hline SR median & & & 0.0 & 3.9 & 53.3 \\
\hline Number of fibers & & & 16 & 15 & 47 \\
\hline Observations & & & 66 & 53 & 211 \\
\hline $\begin{array}{l}\text { Linear regression } \\
\text { result }(r) \text { and statistical } \\
\text { significance }\end{array}$ & $\begin{array}{l}\text { I5 Jitter difference } \\
\text { vs. I3 acoustic rate } \\
\text { (Figs 5A, C, E) } \\
\\
\text { I5 Jitter difference } \\
\text { vs. (I4-I3) acou. rate } \\
\text { diff (Figs. 5B, D, F) }\end{array}$ & $\begin{array}{l}r \\
T \\
p_{\text {error }} \\
p_{\text {error, corrected }} \\
\mathrm{df} \\
r \\
T \\
p_{\text {error }} \\
p_{\text {error, corrected }} \\
\text { df }\end{array}$ & $\begin{array}{l}0.110 \\
0.885 \\
0.190 \\
1.0 \\
64 \\
0.152 \\
1.23 \\
0.111 \\
<0.111 \\
64\end{array}$ & $\begin{array}{l}0.187 \\
1.36 \\
0.090 \\
0.540 \\
51 \\
0.172 \\
1.25 \\
0.109 \\
0.654 \\
51\end{array}$ & $\begin{array}{l}0.247 \\
3.68 \\
0.000145 \\
0.00087 \\
209 \\
0.181 \\
2.66 \\
0.0042 \\
0.0252 \\
258\end{array}$ \\
\hline
\end{tabular}

The jitter plots of Figure 6 were analogous to the spike-rate plots of Figure 5 and the statistical tests summarized in this table are the same as those provided by Table 1. In contrast to the Table 1 results, which indicated significant post-masking spike-rate reductions for all SR groups, the Table 2 test results indicate that postmasking jitter reductions only occurred for the fibers with high spontaneous rates (i.e., SR group 3). Both corrected and uncorrected error probabilities are shown to demonstrate that the correction did not result in false rejections of the hypothesis

spontaneous rate. Individual data from 77 ANFs are plotted. Although the linear correlations (dashed lines in the graphs) produced low correlation coefficients, the slopes of the lines were both significantly lower than zero, using Bonferroni corrected tests (Fig. 7A: $r=0.197$, $t=3.63, n=327$, $p_{\text {error, }}$ corrected $=0.0003$; Fig. 7B: $r=0.185$, $t=3.40, n=327, p_{\text {error, }}$ corrected $=0.00075$ ).

The above analyses suggest that post-masking changes in jitter rely upon spontaneous activity. One additional test was performed to address this question in a relatively simple manner by testing the hypothesis that the interval 5 jitter differences from either the Group 1 or Group 2 fibers were statistically different from zero. The unpaired t-test for the Group 1 jitter differences failed to find a significant difference $(t=$ $\left.0.741, p_{\text {error, uncorrected }}=0.46, \mathrm{df}=130\right)$ whereas the test over the Group 2 data did reach significance $(t=4.92$, $p_{\text {error, }}$ corrected $\left.=0.0066, \mathrm{df}=104\right)$. We conclude that post-masking reductions in jitter occurred in ANFs with spontaneous activity, but were not evident across fibers with negligible $(<0.65$ spike/s) spontaneous activity.

Absolute values of interval 5 jitter While the degree of spike jitter certainly influences ECAP amplitude, the exact relationship between the two measures is not known. As such, jitter ratios or differences may be less revealing than absolute jitter values. Figure 8 plots interval 5 "electric+acoustic" jitter values vs. "electriconly" values from 77 fibers. The data are plotted on linear-linear (Fig. 8A) and $\log -\log$ (Fig. 8B) axes to highlight different ranges and the three SR groups are indicated by symbol color. The diagonal lines indicate equal jitter for the two stimulus conditions. Group 1 jitter values and roughly half of the Group 2 cluster near the equality lines and have values within the range reported for electrically stimulated fibers with no acoustic sensitivity (Miller et al. 1999b). In contrast, the Group 3 jitter values are, with few exceptions, the largest across all three SR groups. These trends again indicate that changes in jitter decrements due to forward masking are strongly dependent on the SR of a fiber.

Changes in mean spike latency In our usage, jitter is a within-fiber statistic estimated through the acquisition of repeated latency measures. As an ECAP is typically acquired by averaging multiple response waveforms, within-fiber jitter can influence the ECAP. However, as jitter is the standard deviation of spike times, it cannot be used to assess across-fiber synchrony, as the latter requires latency measures. We therefore examined how mean spike latency (MSL, the mean latency of spikes acquired through multiple stimulus presentations) was influenced by acoustic masking, SR, and electrically evoked spike rate. Figures 9A-C plot MSL versus spike rate for "electric-only" responses obtained over intervals 1, 2, and 5, respectively, using data from 77 ANFs. Figure 9D plots the same quantities for "electric +acoustic" responses obtained over interval 5. For each plot, the symbol type is used to categorize the data by the three aforementioned SR groups (Group 1: circles, Group 2: squares, Group 3: diamonds). Data from fibers observed to produce a $\beta$ response are indicated by filled (gray) symbols. 

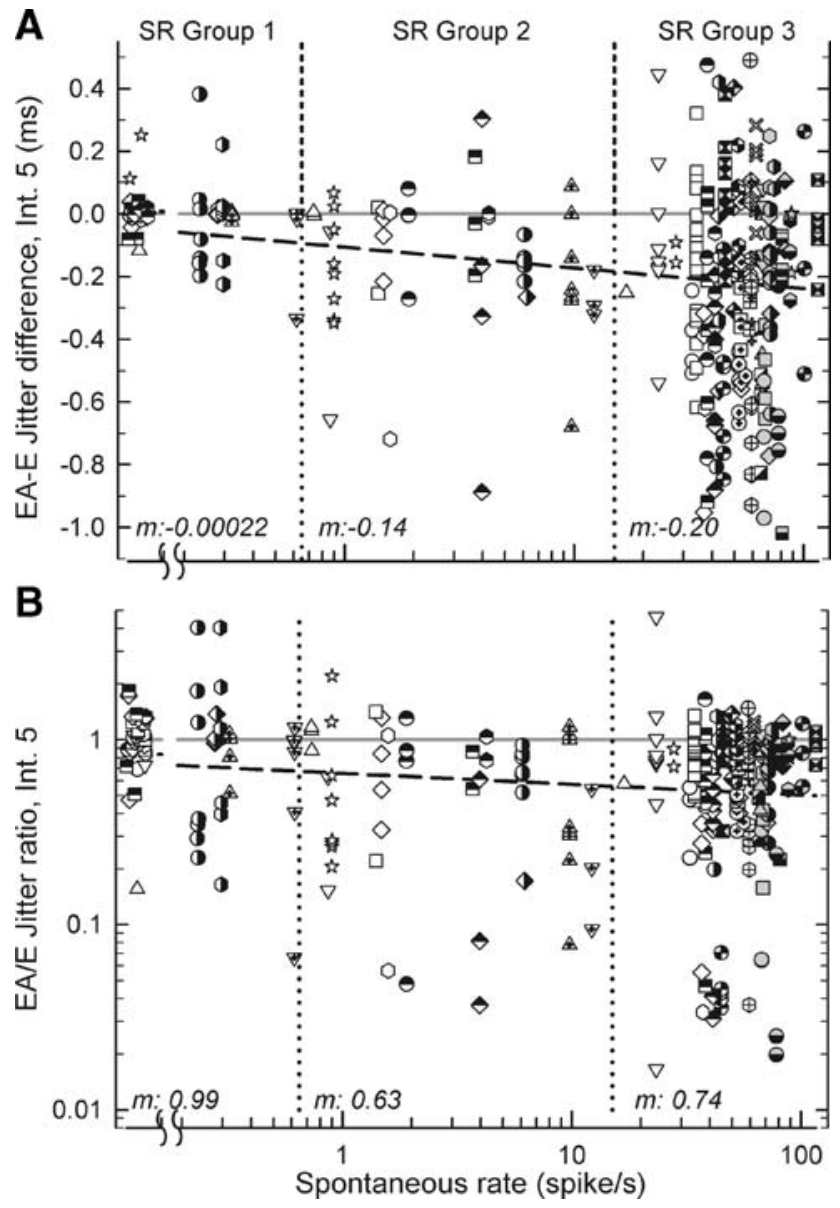

FIG. 7. Additional analyses of interval 5 jitter differences (A) and jitter ratios (B) again indicate that acoustic forward-masking reduces jitter, but those reductions are proportional to the degree of spontaneous activity of the fibers. Data from 77 fibers are shown using a unique symbol for each fiber and the linear correlation coefficients indicate significant functional relationships. Median $($ " $\mathrm{m}$ ") values for each SR group are indicated at the bottom of each plot.

Over interval 1, the data suggest two functional relationships between MSL and spike rate (Fig. 9A). MSLs for Group 1 fibers generally remain constant across response rate and are similar to those produced by deaf ears (Miller et al. 1999b). For ANFs with some spontaneous activity (Groups 1 and 2), MSLs decrease from about $2 \mathrm{~ms}$ to values less than $1 \mathrm{~ms}$ as driven rate increases from 0 to about $150 \mathrm{spike/s}$, a trend consistent with the progressive loss of spontaneous activity. However, some fibers with $\beta$ responses maintain high MSLs for rates between 160 and 300 spike/s, consistent with $\beta$ dominated responses. The only fibers that respond with rates greater than 300 spike/s are those with $\beta$ responses. Spike rates greater than 250 spike/s indicate that each electric pulse evoked, on average, more than one action potential, which explains the trend toward higher MSLs at these highest response rates. The MSL vs. rate trends observed in interval 2 (Fig. 9B) are

largely similar to those of interval 1 , with the exception of the loss of high MSLs for spike rates above $160 \mathrm{spike/s}$. This is consistent with the relatively faster rate of $\beta$-response adaptation reported by Miller et al. (2006). The distribution of MSLs for the electriconly interval 5 is similar to that of interval 2 (Fig. 9C vs. Fig. 9B).

The MSL trends obtained with prior masking (Fig. 9D) differ from those data obtained without

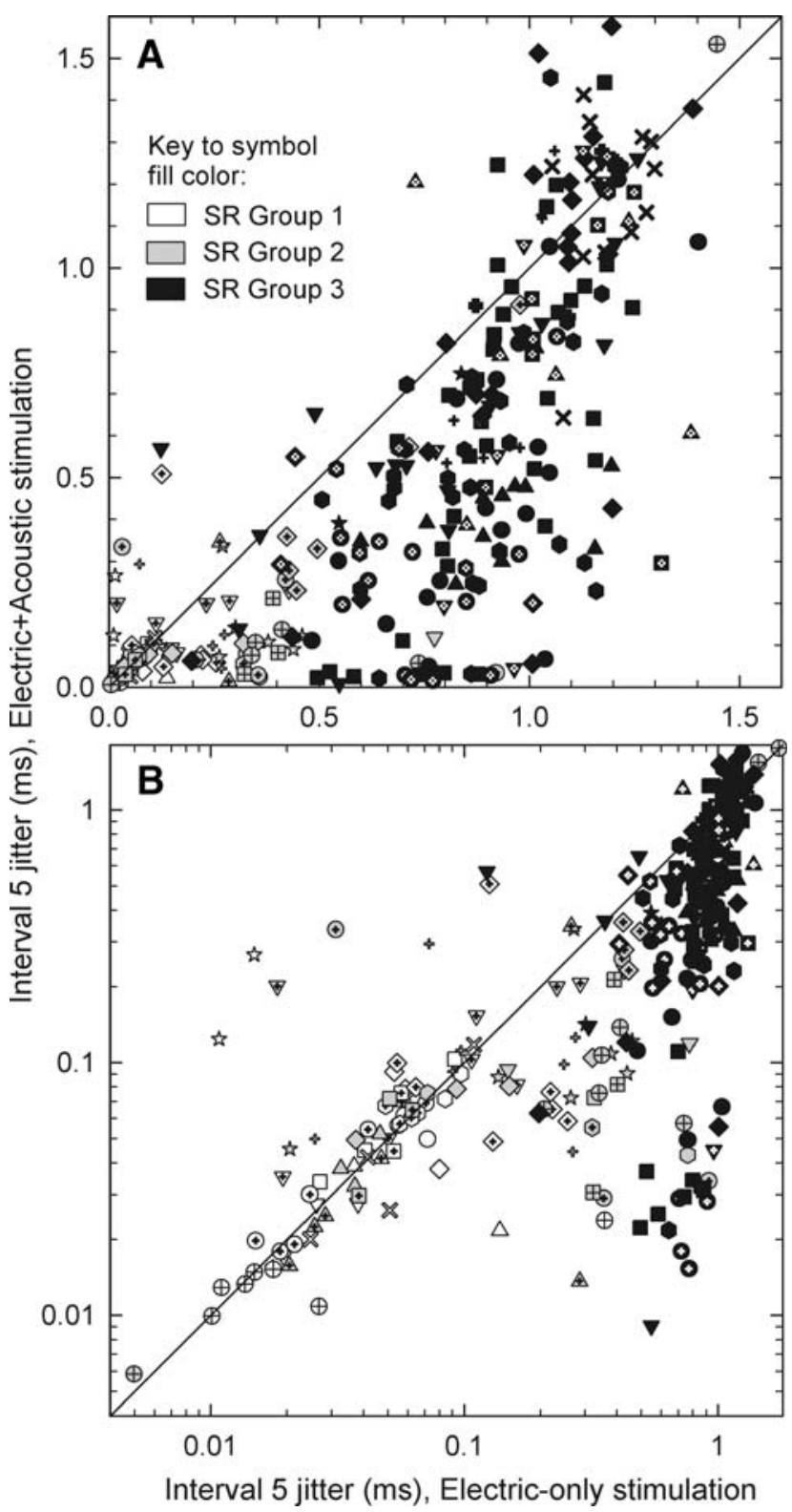

FIG. 8. Absolute jitter values for the "electric+acoustic" postmasking interval, plotted versus the jitter values for the "electriconly", indicate that (1) forward masking reduces jitter for fibers with spontaneous activity, (2) the amount of jitter is proportional to SR, and (3) the jitter values for the Group 1 and some Group 2 fibers are comparable to those obtained from deafened fibers stimulated by single electric pulses (Miller et al. 1999a). The same data are plotted along linear (A) and logarithmic (B) axes. Different symbols identify each ANF and are consistent with those used in Figure 7. 


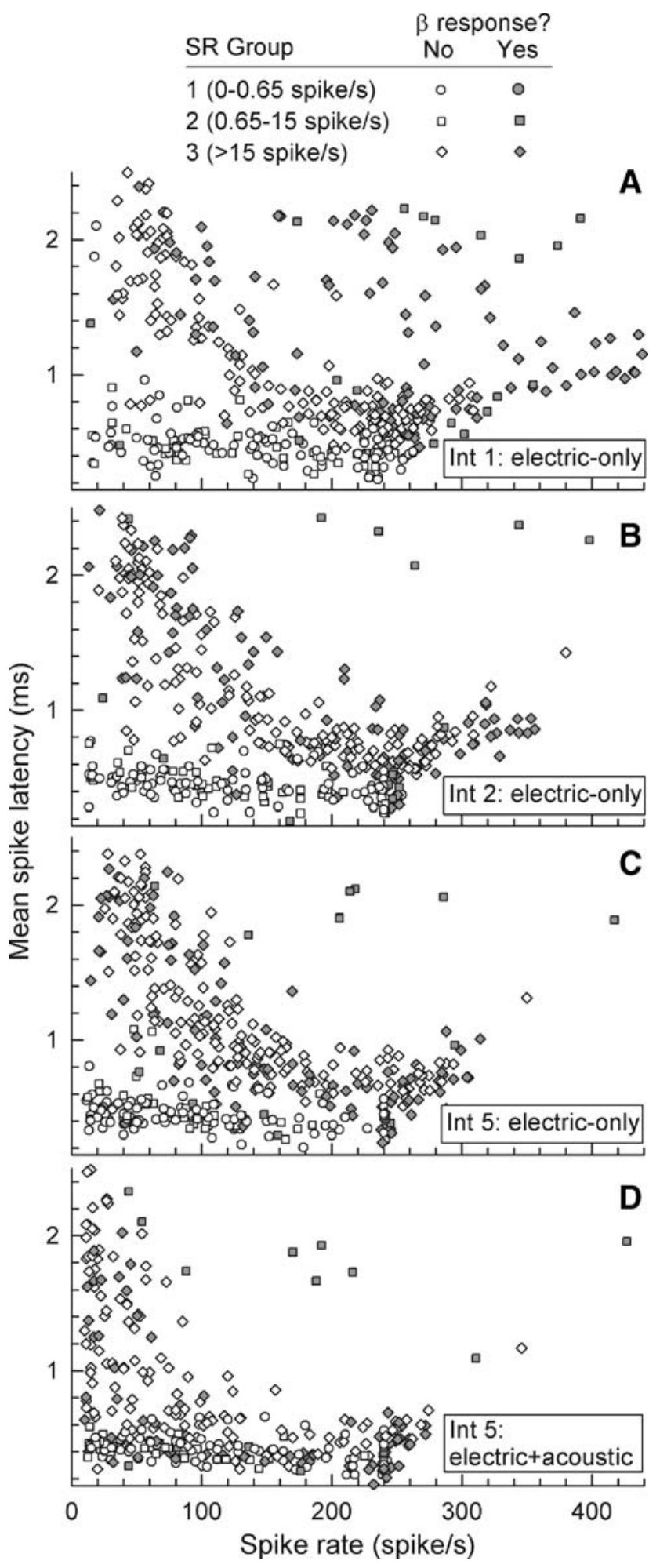

acoustic masking (Fig. 9C), with the former demonstrating relatively precipitous decline in MSLs with increases in spike rate. The differences between these two interval 5 MSL distributions are quantified in Figure 10, which presents MSL box plots across the range of spike rates. The boxes were computed
FIG. 9. The relationship between mean spike latency (MSL) and spike rate varies, to small degrees, across "electric-only" intervals 1 , 2 , and 5 and to a larger degree for "electric+acoustic" responses in post-masker interval 5 . The data, obtained from 77 ANFs, indicate ensemble synchrony. As discussed in the text, some MSL reductions across (A), (B), and (C) likely relate to the loss of $\beta$ responses. The post-masking MSLs (D) have less variability for all but the lowest response rates, due largely to MSL reductions among fibers with spontaneous activity. All graph have the same ordinate range ( 0.15 to $2.5 \mathrm{~ms}$ ) and the presence of electrophonic activity is noted by the dark-gray symbol fills.

across data subsets comprising 40 data samples and are plotted as functions of the median spike rate for each subset. For median spike rates greater or equal to $40 \mathrm{spike/s}$, the "electric+acoustic" box plots indicate narrower MSL distributions than the corresponding "electric-only" MSLs. To summarize the temporal effects, forward masking resulted in less jitter and more uniform MSLs across excited ANFs and those changes were evident in ANFs with high spontaneous activity, but not in fibers without spontaneous activity.

Jitter and rate recovery: use of smaller analysis bins

The six-interval analysis of time-related changes was used to reduce the variability that would result if the response to each electric pulse were examined (a particular problem for measures within interval 5 , due to rate adaptation). The use of shorter, 4-ms analysis windows provides noisy, but detailed temporal descriptions. Figure 11 shows, for fibers from three subjects, rate, and jitter functions using $4 \mathrm{~ms}$ bins for the

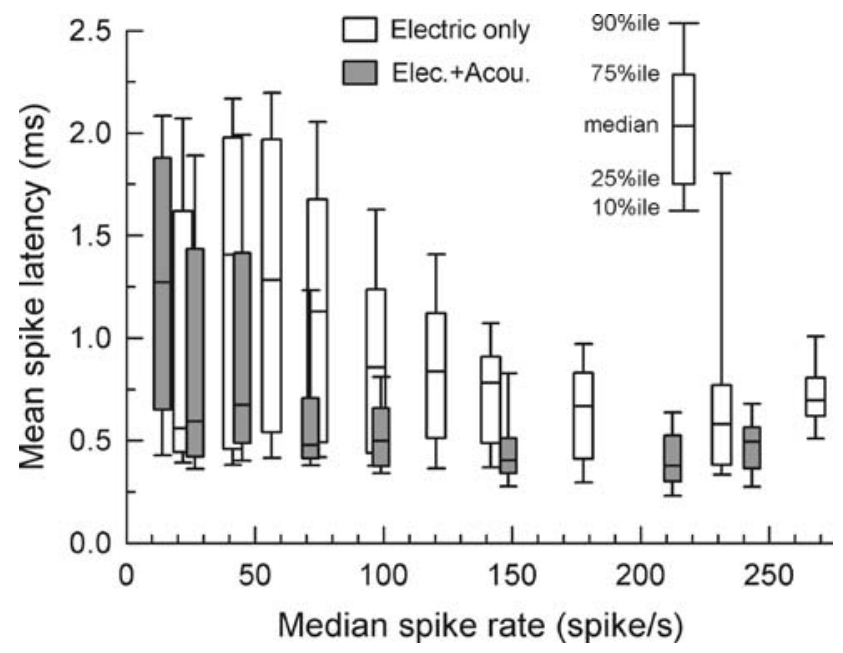

FIG. 10. Box plots quantify the reduction in across-fiber variability in MSL due to forward masking. Interval 5 MSLs are plotted vs. median spike rates for the "electric-only" interval 5 responses (whitefilled boxes) and post-acoustic-masking interval 5 responses (dark gray boxes). 

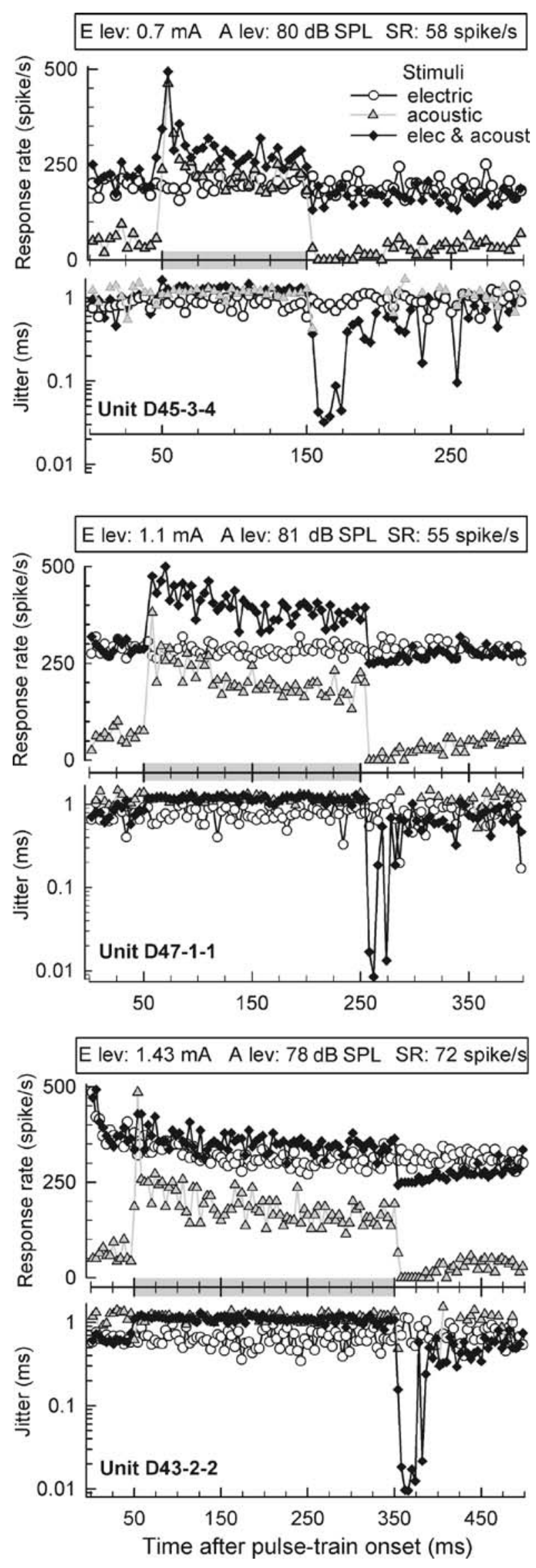

three stimulus conditions. In each case, for electric +acoustic stimulation, jitter dropped precipitously after noise offset and, after about $20 \mathrm{~ms}$, recovered rapidly, followed by slower recovery. Changes in spontaneous activity can be seen in the "acoustic only" plots (i.e., the triangular symbols assume their lowest positions immediately after noise offset). Note that spontaneous activity is absent after noise offset for periods of time similar to those over which jitter is greatly reduced. In contrast, spike rate undergoes a decrement after noise offset, followed by a slower rate of recovery. The data of Figure 11 provide the clearest examples of the differential recovery rates. From visual inspection, we judged that these patterns occurred in about $30 \%$ of the ANFs, although they were not as clear as in the Figure 11 examples. These post-noise recovery patterns were only obvious in fibers with relatively high ( $>15$ spike/s) SRs. As the ECAP depends on both synchrony and response probability, the different time courses of these properties could contribute to the ECAP's non-monotonic post-masking recovery.

\section{Infrequent observation: response enhancement}

We observed increased responsiveness to electric stimuli during the simultaneous presentation of acoustic noise in three cases (i.e., $4 \%$ of studied fibers). The best example of "acoustically enhanced electric responses" produced by a fiber is illustrated in the PSTHs of Figure 12. Histograms for the three stimulus conditions are shown for four electric levels, with the acoustic level fixed at $80 \mathrm{~dB}$ SPL. At the highest electric level used $(1.27 \mathrm{~mA}$, lowest plots in Fig. 12), the electrically evoked FE (assessed over the first $50 \mathrm{~ms}$ of the response) was only $9 \%$. In three of the four sets of PSTHs, the response to the $250 \mathrm{pulse} / \mathrm{s}$ electric train is noticeably increased in the electric+acoustic condition relative to the electriconly condition. The lowest set of PSTHs of Figure 12 includes an inset graph showing the period histogram (based on the electric pulse rate) of the spike activity obtained in the electric-only condition. A bimodal

FIG. 11. Demonstrations of differential rates of recovery of spike jitter and spike rate caused by acoustic forward masking of electric pulse train responses. Data from three fibers are shown. Rate and jitter are plotted versus time, based on the response to each electric pulse, for all three stimulus conditions ("electric-only", "acousticonly", and "electric+acoustic"). Grey bars over the $x$-axes indicate the presentation of the acoustic noise bursts, which are of different durations (100, 200, and $300 \mathrm{~ms})$ for each ANF. In all cases, the electric pulse train was presented across the entire time indicated on each axis. The loss of spontaneous activity following the noise lasts for periods between 20 and $30 \mathrm{~ms}$ (triangle symbols). After masking, electrically evoked jitter plunges to very low values during those same periods (diamond symbols). In contrast, recovery of electrically driven rate follows a slower time course, at least for the two fibers shown in the lower two sets of plots. 

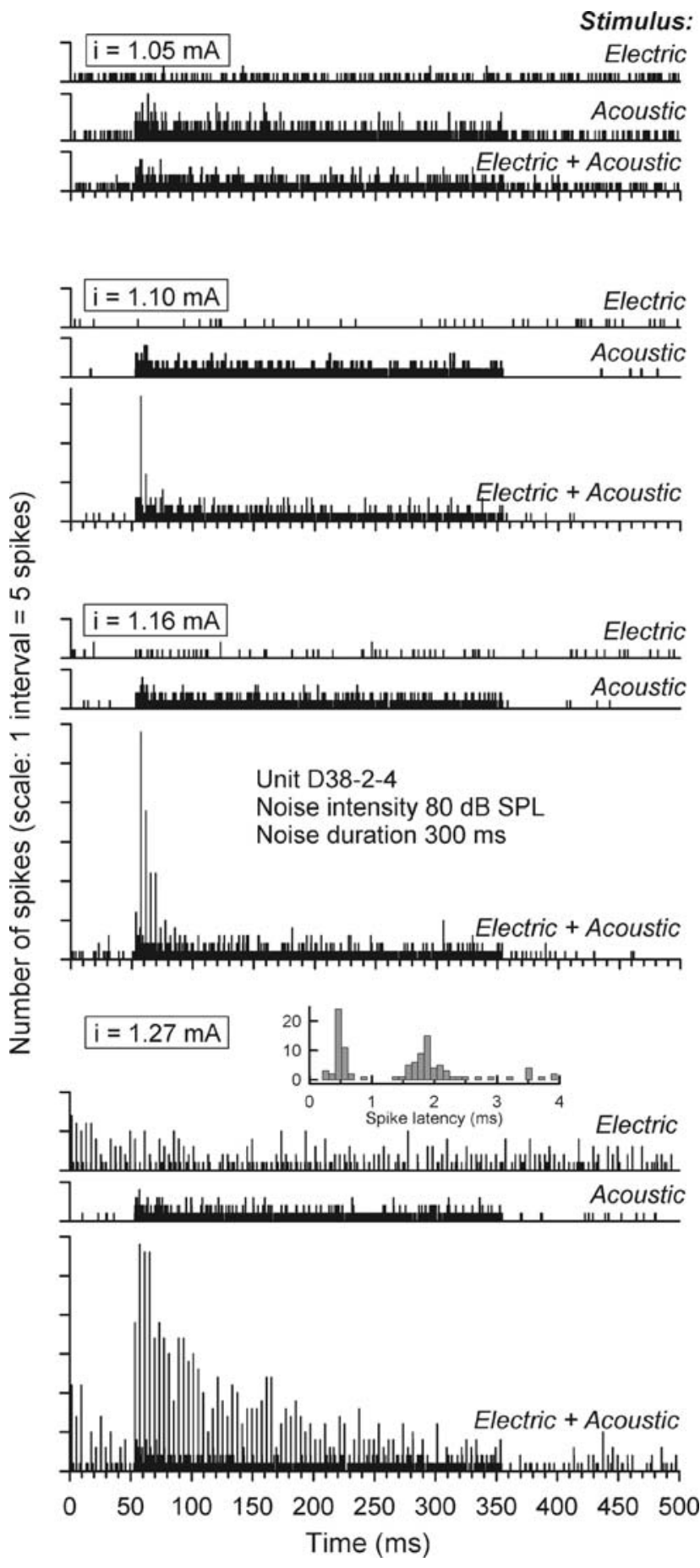

FIG. 12. Demonstration of the infrequently observed phenomenon of enhanced responses to electric pulses during simultaneous presentation of the acoustic noise stimulus. Data are shown for one ANF stimulated at four electric levels. For each level, PSTHs are shown for the "electric-only", "acoustic-only", and the combined "electric+acoustic" stimuli. The small inset graph included with the lowest set of PSTHs shows the period histogram for the electric-alone stimulus condition, indicating two clusters of latencies that correspond to $\alpha$ and $\beta$ responses. distribution of spike times is evident, with the two spiketime distributions corresponding to the $\alpha$ and $\beta$ responses (van den Honert and Stypulkowski 1984). Note that the enhanced electric responses demonstrated rapid rate adaptation relative to adaptation rates observed in other fibers of this study or in deafened, electrically stimulated ANFs (Zhang et al. 2007).

\section{DISCUSSION}

\section{Summary}

We addressed how a generalized acoustic stimulus (noise) interacts with ANF responses evoked by moderate rate electric pulse trains. Most analyses were conducted over time intervals that provided assessment of responses to combined (i.e., simultaneous "electric+acoustic") stimulation and post-acoustic responses. Output measures were often normalized to electric-only response measures and are typically discussed from that perspective. Combined stimulation resulted in greater spike rates in almost all conditions (Figs. 3-A). As the effective electric level was increased, the combined rate typically increased in a nonlinear, compressive, manner (Fig. 4A), although linear addition occurred in specific cases. While increases in the electrically driven rate clearly increased spike rates for combined stimulation (Fig. 3-A), those increases had no noticeable influence on spike jitter. Thus, for simultaneous stimulation, the level-dependent changes in $\mathrm{EA} / \mathrm{E}$ rate ratios (Fig. 3-A) and $\mathrm{EA} / \mathrm{E}$ jitter ratios (Fig. 3-B) were due to different response processes, namely, changes in electric+acoustic rates and changes in electric-only jitter, respectively.

Given the reported changes in forward-masked ECAPs (Nourski et al. 2007), our expectations of forward-masked ANF responses were not clear. Thus, we examined several ANF response properties using a paradigm similar to the ECAP protocol. Post-masking changes in spike amplitude were inconsequential, while relatively large changes in spike rate, jitter, and across-fiber latencies were found. Post-masking effects on spike jitter were evaluated using several approaches. A parsimonious conclusion is that the post-masking changes and their subsequent recovery underlie the reported non-monotonic ECAP recovery functions and that changes in spontaneous activity played a key role.

\section{Effect of driven rates on ANF responses to "combined" stimulation}

Changes in spike rate Electric and acoustic level effects were examined by using the spike rates evoked by each stimulus as the independent variables. This facilitated the combining of data across fibers and 
investigating trends in terms of a functional measure. Electric-level effects on spike rate and jitter were assessed for both combined stimulation and for the post-masking epoch (Fig. 3). Over the range of electrically driven activity explored in this study, the addition of the electric stimulus almost always increased spike rate during combined stimulation (Fig. 3-A and 4A). At relatively high electric levels, EA/E spike-rate ratios approached, but did not reach 1, indicating that the electric stimuli did not completely mask acoustic spike activity during simultaneous stimulation. However, the EA/E spike-rate ratios shown in Figure $4 \mathrm{~A}$ were generally lower than those predicted by linear addition of acoustic and electric activity. For low rates of electrically evoked activity (the left-most portion of Fig. 4A), the data approximated linear addition, probably because low electric rates did not cause significant refractoriness or rate adaptation. The subdivision of data into different ranges of acoustically and electrically evoked rates (Figs. 4A, B) provides a means of assessing the relative impact of changes in acoustic and electric level. The three plots of Figure 4A converge toward a common asymptote, suggesting that electric stimulation dominates the combined (electric+acoustic) response at high levels. This is consistent with the fact that electric stimuli can drive ANFs to higher rates than can acoustic stimuli (Kiang and Moxon 1972).

Changes in jitter While combined stimulation always increased spike rates relative to electric-only stimulation, changes in jitter with combined stimulation were dependent on the degree of electrically driven activity (Fig. 3-B). For relatively low electrically driven rates (i.e., $<100 \mathrm{spike/s}$ ), combined stimulation generally did not change jitter relative to the electric-only measures and were consistent with random spike timing (Figs. 3-C, D) that would result from acoustic-noise stimulation. Thus, for driven rates less than 100 spike/s, electrically driven activity had little apparent influence on spike timing with combined stimulation, even though it clearly increased spike rate (Fig. 3-A). Indeed, for combined stimulation, there was no evident change in jitter across the entire (0-320 spike/s) range of electrically driven rates (Fig. 3-C). Furthermore, the jitter values were characteristic of random spike timing. Thus, leveldependent increases in $\mathrm{EA} / \mathrm{E}$ jitter ratios were due only to decreases in jitter for electric-only stimulation. We suspect that those decreases were due, in part, to the increasing incidence of lower-jitter $\alpha$ responses and fewer high-jitter $\beta$ responses as electric level was increased (van den Honert and Stypulkowski 1984), as well as the reduced influence of spontaneous activity.

The observation that increases in the electrically driven activity had no measurable effect on the timing of action potentials during combined (acoustic+electric) stimulation is perhaps surprising. Increases in electrically induced activity did alter spike timing for the case of electric-only stimulation (Fig. 3-D), resulting in more synchronous firing across a wide range of driven rates. Thus, the influence of electrically driven activity on spike timing is dependent upon the magnitude of "ongoing" spike activity initiated at the hair cell.

\section{Changes in ANF responsiveness following acoustic masking}

A key aspect of this study was to describe how specific ANF response properties (related to electric stimulation) were altered by forward masking by an acoustic stimulus. Jitter measures were preferred over VS, as the latter was somewhat arbitrarily based on the $4 \mathrm{~ms}$ interpulse interval. Decreases in spike rate, jitter, and across-fiber variability in mean latency were observed over the first $20 \mathrm{~ms}$ after the masker offset. Although we did not perform detailed analyses of the rates of recovery of these properties, the across-ANF data trends of Figure 2 suggest that rate reductions, in comparison with jitter changes, were relatively slow to fully recover over the $150 \mathrm{~ms}$ post-masking period. Rate and jitter reductions, no doubt dependent upon relative acoustic and electric levels, could be large. The median spike-rate ratio (measured in analysis interval 5) was 0.61 and one-quarter of the observations had ratios of $25 \%$ or less. The median jitter ratio was 0.80 , with one-quarter of the observations with ratios of $45 \%$ of less. The spread of mean spike latencies (assessed over the two middle quartiles) were reduced twofold over a considerable range of firing rates (Fig. 10) and those changes typically occurred within fibers with significant spontaneous activity (Fig. 9C versus Fig. 9D).

Our use of a $20 \mathrm{~ms}$ post-masker analysis window likely obscured the maximal effects of the acoustic masker. A comparison of the post-masking jitter changes obtained with the six-window analyses (Fig. 2) against those evident with a finer temporal analysis (Fig. 11) underscores this point. Avery small (on average, $1 \%$ ) decrease in spike amplitude was observed across the masker duration. Using a single electric masking pulse, much larger decrements (up to $40 \%$ ) can be observed over a relatively brief (i.e., $1 \mathrm{~ms}$ ) epoch following the masker (Miller et al. 2001). It is also likely that our stimulus paradigm, which provided only a $1 \mathrm{~s}$ dead time between each of the three stimulus conditions, resulted in some long-term adaptation that may have reduced the magnitude of post-masking effects. This may not greatly handicap the utility of our findings relative to clinical studies, as the auditory nerves of implant users can be subjected to a degree of "ongoing" stimulation.

Clearly, however, our paradigm captured effects of forward masking. The duration of the masker (100- 
$300 \mathrm{~ms}$ ) was within the range of so-called short-term adaptation (Smith 1977) over which rate and SR reductions occur. However, the increases in withinand across-fiber synchrony (i.e., jitter and MSL) measured in response to electric stimuli have not been previously described. The plots of Figures 5 and 6 demonstrated that post-masking jitter and spike-rate changes were correlated to both the initial acoustically driven rate and the rate reduction observed across the masker's duration. The scatter plots contain too much variability to determine whether or not the relationships between the dependent and independent variables differ from the simple assumption of linear relationships. However, the fact that both measures (initial rate and rate decrement) associated with the acoustic response correlate to post-masking effects is consistent with the temporal pattern of acoustically induced adaptation. Specifically, the onset rate in response to an acoustic masker is proportional to the rate decrement observed across the duration of the masker (Smith and Zwislocki 1975).

\section{The influence of spontaneous activity}

This study was motivated, in part, by the possibility that changes in spontaneous activity were related to the aforementioned ANF temporal response changes. We therefore conducted multiple analyses of jitter changes in the post-masking interval that (1) separately considered each of three SR groups (Figs. 6, Table 2, and Fig. 8), (2) examined linear regression over SR (Fig. 7), and (3) used relatively simple $t$-tests. The analyses indicated that post-masking reductions in jitter were always associated with ANFs having some degree of spontaneous activity. Fibers with the greatest SRs exhibited the largest jitter reductions, while reductions were not observed among the Group 1, "very low SR", fibers. Also, the reduction in the acrossfiber variance in spike latency (Figs. 9 and 10) were due to shifts in the latency distributions of ANFs with appreciable spontaneous activity. We conclude that the post-masking changes in jitter and MSL were due to transient reductions in spontaneous activity. Reductions in SR would reduce degree of refractoriness, thereby reducing temporal uncertainty (jitter) and increasing across-fiber synchrony (i.e., reducing ensemble MSL variability).

\section{Relationship with ECAP recovery}

The time courses of the ANF effects are generally consistent with the ECAP changes reported by Nourski et al. (2007). That is, large effects were observed within $20 \mathrm{~ms}$ after masker offset and response properties were largely recovered to baseline values within 100-200 ms. To the extent possible with our analyses, the ANF temporal and rate properties were observed to recover monotonically after masker offset. While post-masking rate reductions would reduce the ECAP, decreases in jitter and MSL variability (i.e., increases in within- and across-fiber synchrony) would be expected to increase the ECAP. These post-masking changes in ANF response properties provide the basis for non-monotonic ECAP recovery. We also noted that post-masking rate decrements increased as noise duration was increased from 100 to $300 \mathrm{~ms}$. However, additional decreases in jitter were not observed. This difference in the sensitivity of postmasking rate and jitter changes would be expected to influence the degree of non-monotonicity in ECAP recovery and is consistent with duration-dependent changes in ECAP recovery (Nourski et al. 2007, Fig. 5).

While it is plausible that ECAP amplitude is linearly related to ensemble spike rate, the influence of jitter and across-fiber MSL variability are not obvious. It would be expected that, as jitter (or across-fiber latency variability) is increased from small values relative to the width of an action-potential, its influence on ECAP decrements would increase. This was demonstrated in the phenomenological model of Miller et al. (1999b), which could vary jitter independently of other properties to assess its effect on ECAP growth functions (Fig. 11 of that paper). The model predictions suggest that small (i.e., $0.05 \mathrm{~ms}$ ) deviations in jitter away from the nominal, $0.1 \mathrm{~ms}$ value (typical of cat ANFs at $50 \%$ firing efficiency) cause relatively small changes in ECAP amplitude. In contrast, the same deviations about larger jitter values result in greater ECAP changes. As jitter is increased beyond the width of the action potential $(\sim 1 \mathrm{~ms})$, ECAP amplitude would be expected to decrease toward negligible values. Low-SR fibers typically had low jitter that did not change with forward masking (Fig. 8), while high-SR fibers could exhibit large decreases (Figs. 8 and 11) that would be expected to increase the ECAP.

If post-masking recovery of jitter, MSL, and spike rate occurred at markedly different rates, it could be stated with relative confidence that non-monotonic ECAP recovery would result. The fine-bin analyses of post-masking effects (Fig. 11) indicates that jitter can undergo fast and precipitous reductions, followed by equally fast recovery. In two of the three fibers described in that figure, rate recovery was much slower. The combined effects of these post-masking changes could result in a brief period of ECAPamplitude "enhancement", followed by a reduction (caused by fast jitter recovery) and a final, slower period over which ECAP recovers to its baseline value. Our data identifies the specific ANF response properties that likely underlie non-monotonic ECAP recovery. Follow-up studies using a computational model 
could quantify how incremental changes in each of those properties contributes to ECAP amplitude changes.

A complication to this interpretation is that Nourski et al. (2007) used an electric forward masker (a $5,000 \mathrm{pulse} / \mathrm{s}$ train) with guinea pigs having no measurable acoustic sensitivity and reported nonmonotonic ECAP recovery. Among the animals with which electric maskers were used, four were subjected to intravenous injections of furosemide and three were treated with intracochlear neomycin to effect large acoustic threshold shifts as assessed by the CAP. All four of the furosemide-treated animals demonstrated nonmonotonic ECAP functions prior to furosemide injection. During the furosemide effect (which increased click-evoked CAP thresholds by at least $60 \mathrm{~dB}$ ), the non-monotonic component of the ECAP recovery was abolished in one animal and reduced in the other three. Of the three neomycin-treated animals, the magnitude of the non-monotonic portion of the recovery function was similar before and after treatment in one case, reduced by about $67 \%$ in other, and abolished in one case. These results suggest that nonmonotonic ECAP recovery was partially dependent on acoustic sensitivity in most (five of seven) cases and was totally dependent on acoustic sensitivity in the two other cases.

Under the assumption that the drug treatments largely abolished spontaneous activity, the ECAP data suggest that a second mechanism, one not reliant on spontaneous activity, contributes to increased withinand across-fiber synchrony after forward masking. The results of our study were limited to animals with acoustic sensitivity and cannot address this issue. It should be noted that, relative to the use of an acoustic masker, the electric pulse-train masker of the Nourski et al. (2007) study could have markedly different effects on ANFs and their responses. While both acoustic and electric maskers can change responsiveness due to the spike activity they elicit (i.e., rate adaptation), the effect of electric stimuli can differ from that of acoustic stimuli in two fundamental ways. First, electric stimuli can evoke much greater spike rates than can acoustic stimuli (Kiang and Moxon 1972) thereby causing more profound adaptation effects. Second, electric stimuli change the electrical characteristics of the ANF membrane irrespective of action potential initiation. One possible clue to the post-masking ECAP results obtained using electric maskers is provided by the Miller et al. (2001) study, which used single electric masker pulses to put deafened fibers in refractory states. It was noted that in the short refractory period, spike jitter was reduced relative to the unmasked levels. Transient jitter reduction would tend to increase ECAP amplitude.
It is also possible that the furosemide or neomycin treated animals of the Nourski et al. (2007) study retained a significant amount of spontaneous activity, as this quantity was not directly measured in that study. While furosemide does cause a large, transient, loss of acoustic sensitivity, the sensitivity of SR to furosemide can vary significantly across fibers (Sewell 1984). The ECAP-based experiments conducted by Nourski et al. (2007) cannot rule out the possibility that a presumably low level of spontaneous activity remained in their furosemide or neomycin treated animals. From our single-fiber data, we conclude that post-masking changes in spontaneous activity, which give rise to changes in ANF temporal properties, are required for non-monotonic ECAP recovery.

\section{"Acoustic enhancement" of the electric response}

In $4 \%$ of ANFs examined, simultaneous presentation of acoustic noise resulted in increased spike activity to the electric stimulus (Fig. 12). We speculate that the $\beta$ response, together with acoustic excitation of the basilar membrane, was responsible for the acoustically enhanced response. The responses shown in Figure 12 were produced by an ANF with $\beta$ responses and the relatively fast rate of adaptation of the enhanced responses are consistent with the rate of adaptation of $\beta$ responses (Miller et al. 2006). Moxon (1971) demonstrated that acoustic tones and electric sinusoids could result in responses indicative of the addition of the effects of each stimulus and that this additivity relied on the $\beta$ response. However, the responses we reported are truly "enhancements" in the sense that the addition of the two stimuli did not produce a proportionally greater response, but greater-than-linear addition. Our data cannot identify the mechanism with a certainty; however, such non-linearity is observed in the "stochastic resonance" phenomenon in which the addition of random, noise-like, energy non-linearly increases the response to a deterministic stimulus (Morse and Evans 1996). In the case described here, the noise component of a possible stochastic resonance effect is not dependent on the use of a noisy stimulus (i.e., acoustic noise), but rather on the observation that ANF responses to acoustic stimuli are inherently stochastic (e.g., Kiang and Moxon 1972).

The low reported incidence of this enhancement effect may have resulted from the experimental design. We did not manipulate acoustic and electric levels to maximize the probability of electrophonic responses or optimize acoustic levels for the production of stochastic resonance, an effect which occurs over a relatively narrow range of "near threshold" stimulus levels. Thus, the incidence of enhanced electric responses through a stochastic resonance mechanism may be different than that represented 
in our data set. However, if our supposition regarding this origin of the enhanced response is correct, only those fibers having $\beta$ responses (Stypulkowski and van den Honert 1984) will produce this effect.

\section{Clinical implications}

As in the case of results reported by von Ilberg et al. (1999), this study has shown that combined electric and acoustic stimulation of ANFs can produce responses that can be largely predicted by the "acoustic-only" and "electric-only" responses. Specifically, the spike rates produced by each stimulus are additive for low evoked rates that do not result in appreciable refractory or adaptation effects. For most evoked rates, however, the response to the combined stimuli falls short of linear addition. Our results also indicate that, while the combined spike rate is strongly related to the degree of electrically driven spike activity, the ability of the electric stimulus to entrain spikes to the electric pulses is dependent on the degree of spike activity initiated at the hair cell. For relatively high acoustic levels examined in this report, the addition of electric pulse-train energy did not appear to reduce temporal uncertainty. In contrast, at lower levels of acoustic excitation, electric stimuli can increase spike rate and spike synchrony. Thus, the changes in spike rate and spike timing caused by combined stimulation may not be strongly correlated for some levels of electric and acoustic stimuli and may be more strongly linked for other combinations of stimulus levels.

Thus, the design of future speech-processor/hearingaid combinations that could simultaneously (electrically and acoustically) excite some auditory-nerve fibers may require consideration of a complex set of possible stimulus interactions. In addition to those described in our study, strong interactions can occur via the electrophonic mechanism (Moxon 1971). However, histological data from animals implanted with an intracochlear array indicate that hair-cell survival is restricted to regions apical to the array (Shepherd et al. 1995). Also, consideration of audiometric data from "hybrid" users (e.g., Kiefer et al. 2005) and estimates of the tonotopic position of the apical end of fully implanted electrode arrays (Skinner et al. 2002) suggest it is not likely that implanted arrays appreciably overlap the cochlear region with acoustic sensitivity in "hybrid" implantees. Thus, we may not expect, from the present cohort of "hybrid" subjects, significant degrees of within-ANF acoustic-electric interactions.

Of course, the assumption of little or no overlap of acoustically and electrically excited populations of ANFs is premised upon the notion that electric excitation of the auditory nerve is limited to ANFs with cochleotopic origins near the positions of the intracochlear electrodes. It is possible that "ectopic" excitation of ANFs may occur as a result of modiolar sites of action potential initiation and that interactions such as those described here may occur in "hybrid" ears. Nonetheless, as individuals with wider ranges of hearing are implanted and with closer apical-to-basal positioning of the intracochlear array to the acoustically sensitive region of the cochlea, it is more likely that within-fiber interactions will occur, increasing the relevance of our findings. As noted above, within-fiber interactions of acoustically and electrically driven activity can result in spike rates and temporal firing patterns that are highly dependent upon the relative effective levels of the electric and acoustic stimuli.

Acoustic maskers as brief as $100 \mathrm{~ms}$ could cause non-monotonic ECAP recovery (Nourski et al. 2007) as well as the changes in jitter, spike latency, and spike rate documented here. Available data indicate that the time period over which ECAP amplitudes were increased over baseline values was brief, covering about a 50-ms epoch after the masker offset. Grosspotential increases and non-monotonic recovery are unique to electrically evoked responses following masking and would presumably be relevant to stimulus coding by ANFs responsive to both stimulus types. Such effects could clearly alter the representation of electrically coded acoustic information processed by an auditory prosthesis. While the duration of the postmasking effects may be short, their magnitudes can be appreciable. Abrupt changes in across-fiber synchrony could be expected to affect stimulus coding and subsequent processing of eighth-nerve output by central auditory neural centers.

It can be appreciated that computational modeling of auditory nerve responses may prove useful, as well as relatively efficient, in describing how various realworld stimuli may interact. As non-monotonic ECAP recovery (and hence, presumably changes in ANF jitter and across-fiber synchrony) can occur in deaf ears with electric stimulation, such a model would not necessarily have to incorporate cochlear mechanics or hair-cell transduction elements, but may require mechanisms that account for both refractoriness and longer-term effects typically referred to as adaptation.

\section{ACKNOWLEDGEMENT}

This work was supported by National Institute on Deafness and Other Communication Disorders Contract N01-DC-21005 and grant R01-DC006478.

\section{REFERENCES}

Bevington PR, Robinson DK. Data reduction and error analysis for the physical sciences. New York, McGraw-Hill, 2002. 
Cullen RD, Higgins C, Buss E, Clark M, Pillsbury HC 3rd, Buchman CA. Cochlear implantation in patients with substantial residual hearing. Laryngoscope 114:2218-2223, 2004.

Gantz BJ, Turner CW. Combining acoustic and electrical hearing. Laryngoscope 113:1726-1730, 2003.

James C, Albegger K, Battmer R, Burdo S, Deggouj N, Deguine O, Dillier N, Gersdorff M, Laszig R, Lenarz T, Rodriguez MM, Mondain M, Offeciers E, Macias AR, Ramsden R, Sterkers O, Von Wallenberg E, Weber B, Fraysse B. Preservation of residual hearing with cochlear implantation: how and why. Acta Otolaryngol. 125:481-491, 2005.

JAVEL E, SHEPHERD RK. Electrical stimulation of the auditory nerve. III. Response initiation sites and temporal fine structure. Hear. Res. 140:45-76, 2000.

Kiang NY, Watanabe T, Thomas EC, Clark LF. Discharge patterns of single fibers in the cat's auditory nerve. Cambridge, MIT, 1965.

KIANG NY, Moxon EC. Physiological considerations in artificial stimulation of the inner ear. Ann. Otol. Rhinol. Laryngol. 81:714-730, 1972.

Kiefer J, von Ilberg C, Reimer B, Knecht R, Gall V, Diller G, Sturzebecher E, Pfennigdorff T, Spelsberg A. Results of cochlear implantation in patients with severe to profound hearing lossimplications for patient selection. Audiology 37:382-395, 1998.

Kiefer J, Pok M, Adunka O, Sturzebecher E, Baumgartner W, Schmidt M, Tillein J, Ye Q, Gstoettner W. Combined electric and acoustic stimulation of the auditory system: results of a clinical study. Audiol. Neurol. 10:134-144, 2005.

Kong Y-Y, STICKNey GS, Zeng F-G. Speech and melody recognition in binaurally combined acoustic and electric hearing. J. Acoust. Soc. Am. 117:1351-1361, 2005.

Miller CA, Abbas PJ, Robinson BK, Rubinstein JT, Matsuoka AJ. Electrically evoked single-fiber action potentials from cat: responses to monopolar, monophasic stimulation. Hear. Res. 130:197-218, 1999a.

Miller CA, Abbas PJ, Rubinstein JT. An empirically based model of the electrically evoked compound action potential. Hear. Res. 135:1-18, $1999 \mathrm{~b}$.

Miller CA, Abbas PJ, Robinson BK. Response properties of the refractory auditory nerve fiber. J. Assoc. Res. Otolaryngol. 2:216232, 2001.

Miller CA, Abbas PJ, Robinson BK, Nourski KV, Zhang F, Jeng F-C. Electrical excitation of the acoustically sensitive auditory nerve: single-fiber responses to electric pulse trains. JARO 7:195-210, 2006.

Morse RP, Evans EF. Enhancement of vowel coding for cochlear implants by addition of noise. Nature Med. 2:929-932, 1996.
Moxon EC. Neural and mechanical responses to electric stimulation of the cat's inner ear. Massachusetts Institute of Technology Dept of Electrical Engineering Ph.D. Thesis, 1971.

Nourski KV, Abbas PJ, Miller CA, Robinson BK, Jeng FC. Acousticelectric interactions in the guinea pig auditory nerve: simultaneous and forward masking of the electrically evoked compound action potential. Hear. Res. 232:87-103, 2007.

SEWELL WF. The relation between the endocochlear potential and spontaneous activity in auditory nerve fibres of the cat. J. Physiol. 347:685-696, 1984.

Shepherd RK, Clark GM, Xu SA, Pyman BC. Cochlear pathology following reimplantation of a multichannel scala tympani electrode array in the macaque. Am. J. Otol. 16:186-199, 1995.

Skinner MW, Ketten DR, Holden LK, Harding GW, Smith PG, Gates GA, Neely JG, Kletzker GR, Brunsden B, Blocker B. CT-derived estimation of cochlear morphology and electrode array position in relation to word recognition in Nucleus-22 recipients. J. Assoc. Res. Otolaryngol. 3:332-350, 2002.

SмITH RL. Short-term adaptation in single auditory nerve fibers: some poststimulatory effects. J. Neurophysiol. 40:1098-1111, 1977.

Sмiтh RL, Zwislocki JJ. Short-term adaptation and incremental responses of single auditory nerve fibers. Biol. Cybern. 17:169$182,1975$.

SNyder RL, Bierer JA, Middlebrooks JC. Topographic spread of inferior colliculus activation in response to acoustic and intracochlear electric stimulation. JARO 5:305-322, 2004.

Stakhovskaya O, Schoenecker1 MC, Snyder RL, Bonham BH. Topographic differences in acoustic forward masking patterns in the inferior colliculus central nucleus (ICC) of the guinea pig. Assoc. Res. Otolaryngol. Abs. P. 114, 2008.

Stypulkowski PH, van den Honert C. Physiological properties of the electrically stimulated auditory nerve. I. Compound action potential recordings. Hear. Res. 14:205-223, 1984.

van den Honert C, Stypulkowski PH. Physiological properties of the electrically stimulated auditory nerve. II. Single fiber recordings. Hear. Res. 14:225-243, 1984.

von Ilberg C, Kiefer J, Tillein J, Pfenningdorff T, Hartmann R, Sturzebecher E, KLinke R. Electric-acoustic stimulation of the auditory system. New technology for severe hearing loss. ORL J. Otorhinolaryngol. Relat. Spec. 61:334-340, 1999.

Zhang F, Miller CA, Robinson BK, Abbas PJ, Hu N. Changes across time in spike rate and spike amplitude of auditory nerve fibers stimulated by electric pulse trains. J. Assoc. Res. Otol. 8:356-372, 2007. 